\title{
ABSTRACTS
}

\section{The Canadian Coalition for Seniors' Mental Health (CCSMH) and Canadian Academy of Geriatric Psychiatry (CAGP) Joint Scientific Meeting}

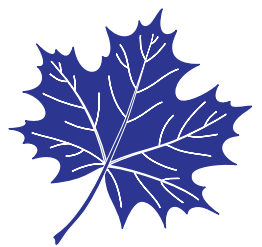

\section{KEYNOTE SPEAKERS}

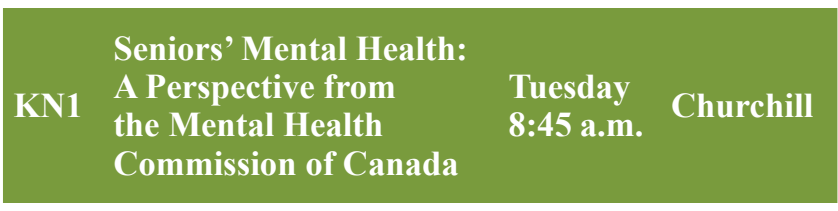

Dr. David Goldbloom, Professor of Psychiatry at University of Toronto, and Chair of the Mental Health Commission of Canada

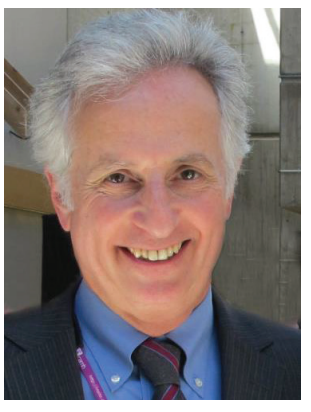

BIOGRAPHY: Dr. David S. Goldbloom was born in Montreal and raised in Quebec and Nova Scotia. He completed an honours degree, majoring in Government, at Harvard University and then attended the University of Oxford as a Rhodes Scholar where he obtained an M.A. in Physiological Sciences. He trained in medicine and psychiatry at McGill University and is a Professor of Psychiatry at the University of Toronto. Dr. Goldbloom's activities have been recognized and awarded by his peers and students. $\mathrm{He}$ has authored numerous scientific articles and book chapters and has provided talks and lectures to student, professional, and public audiences. He maintains an active clinical and teaching role at the Centre for Addiction and Mental Health where he serves as Senior Medical Advisor. He is also Chair of the Mental Health Commission of Canada. In addition to his professional activities, Dr. Goldbloom is the immediate past Chair of the Board of Governors of the Stratford Shakespeare Festival of Canada.

\section{Learning Objectives:}

1. To become familiar with the mission, structure, and work of the Mental Health Commission of Canada (MHCC).

2. To understand the specific initiatives of the MHCC with regard to seniors.

3. To consider how attendees can contribute to these mental health reform efforts.

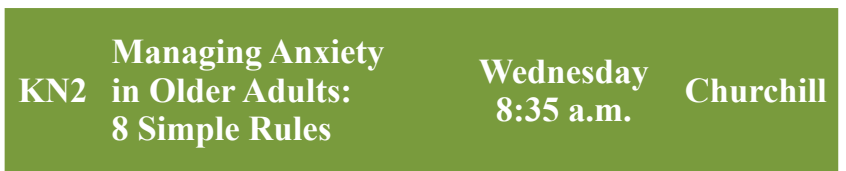

Dr. Eric Lenze, Professor of Psychiatry at Washington University in St. Louis, and leading expert in Late-Life Anxiety Disorders

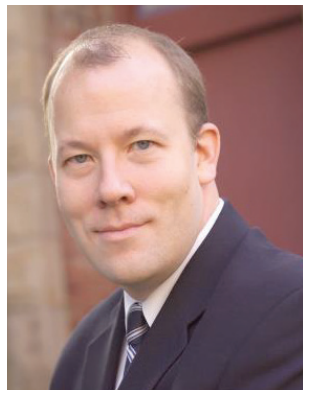

BIOGRAPHY: Dr. Lenze is Professor in the Department of Psychiatry at Washington University School of Medicine. Dr. Lenze completed medical school and psychiatry residency at Washington University School of Medicine. He trained in a geriatric psychiatry fellowship at University of Pittsburgh, and then underwent a research fellowship at the Late-life mood disorders center at University of Pittsburgh.

Dr. Lenze's research focuses on treatment development and testing in anxiety disorders and depression. He has led numerous clinical trials and treatment development studies of pharmacological and behavioral treatments for depression and anxiety disorders. He is author on over 130 peer-reviewed articles, reviews, and book chapters. Dr. Lenze is also a practicing geriatric psychiatrist who focuses on the management of anxious and depressed patients.

\section{PLENARY SPEAKERS}

$\begin{array}{ll}\text { PS1 Training Caregivers of } & \text { Tuesday } \\ \text { Patients with Dementia } & \text { 1:00 p.m. }\end{array}$ Churchill

Dr. Joel Sadavoy, Professor of Psychiatry, University of Toronto, Inaugural CAGP President

BIOGRAPHY: Dr. Joel Sadavoy is professor of psychiatry and member of the Institute of Medical Sciences and Neurosciences program at the University of Toronto. He is head of Geriatric and Ethno-cultural Community Psychiatry, and founder and 
Director of the Reitman Centre for Alzheimer's Support and Training at Mount Sinai Hospital where he is the inaugural holder of the Pencer Family Chair in Applied General Psychiatry and the immediate past Psychiatrist-in-Chief. His key academic interests have been in service development research and implementation, most recently in the area of family caregivers of persons with dementia, ethno-cultural psychiatry, and psychotherapy of the elderly. He is the founding past president of the Canadian Academy of Geriatric Psychiatry, and a past president of the International Psychogeriatric Association.

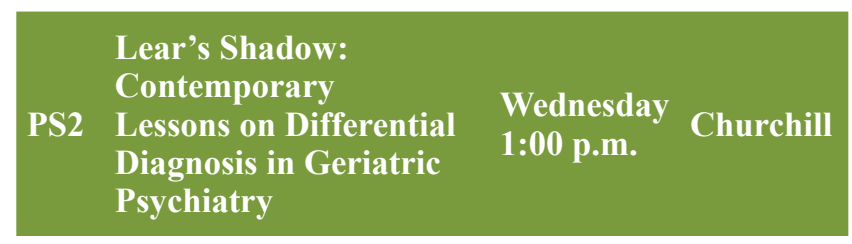

Dr. Mark Rapoport, Associate Professor of Psychiatry at University of Toronto, and President, Canadian Academy of Geriatric Psychiatry

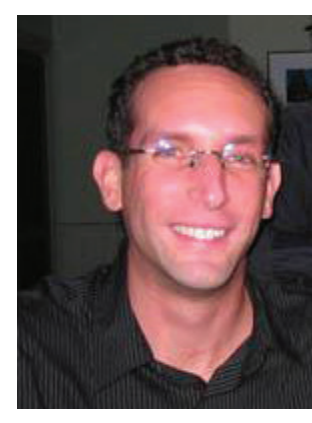

BIOGRAPHY: Dr. Rapoport is an associate professor in the department of psychiatry at the University of Toronto, and is an associate scientist at Sunnybrook Research Institute. He is also a member of the Canadian Driving Research Initiative for Vehicular Safety in the Elderly (CanDRIVE), and an associate editor of the Canadian Journal of Geriatrics. Dr. Rapoport received his medical degree from McMaster University in 1995, and completed his residency and fellowship in psychiatry at the University of Toronto, where he now works as a geriatric neuropsychiatrist. He has been a board member of the Canadian Academy of Geriatric Psychiatry (CAGP) since 2006, cochairing their annual scientific meetings, and was the scientific co-chair for the 2009 International Psychogeriatric Association Congress. His main areas of research are traumatic brain injury in the elderly and the risk of motor vehicle crashes associated with neurological and psychiatric diseases and their treatments.

\section{SYMPOSIUM ABSTRACTS}

\begin{tabular}{|c|c|c|}
\hline S1 & $\begin{array}{l}\text { Novel Biomarker } \\
\text { Development for Use in } \\
\text { Clinical Interventions }\end{array}$ & $\begin{array}{l}\text { Tuesday } \\
\text { 10:30 a.m. }\end{array}$ \\
\hline
\end{tabular}

Aristotle Voineskos, MD, PhD, FRCPC ${ }^{1}$, Daniel M. Blumberger, MD, MSc, FRCPC ${ }^{2}$, Tarek K. Rajji, MD $^{3}$, Benoit H. Mulsant, MD, $\mathrm{MS}^{3}$ ${ }^{1}$ Kimel Family Translational Imaging-Genetics Laboratory, Centre for Addiction and Mental Health; ${ }^{2}$ Late-Life Mood Disorders Clinic, Centre for Addiction and Mental Health; ${ }^{3}$ Campbell Family Mental Health Research Institute, Centre for Addiction and Mental Health and University of Toronto
This symposium application highlights the development of novel, cutting-edge biomarkers using neurophysiological and neuroimaging approaches that are relevant for geriatric populations. The presenters will show how these novel biomarkers have been developed in older populations. The practical utility of these biomarkers to track symptom or disease progression, understand treatment effects, and predict treatment response will also be highlighted.

First, Dr. Daniel Blumberger will introduce transcranial magnetic stimulation (TMS), which is a non-invasive form of brain stimulation that capitalizes on the ability of time-varying magnetic fields to induce electrical currents in biological tissue via the principle of electromagnetic induction. TMS can measure dysfunctional cortical inhibition (CI) and neuroplasticity, which have been postulated as a neurophysiological mechanisms involved in treatment resistant depression (TRD). These paradigms also have potential to predict response to existing psychopharmacological treatments. Neurophysiological data from three groups will be presented (i.e., from non-depressed older adults, depressed older adults without treatment resistance and depressed older adults with treatment resistance.) Neurophysiological predictors of response to treatment will also be shown, highlighting the utility of TMS neurophysiology paradigms as biological predictors of treatment response and illness severity.

Second, Dr. Tarek Rajji will discuss deficits in neuroplasticity in patients with schizophrenia across the adult lifespan. Deficits in working memory performance are a core feature of people with schizophrenia across adult life. Modulation of gamma oscillations amplitude by theta oscillations phase ("theta-gamma coupling") is thought to mediate working memory in the dorsolateral prefrontal cortex (DLPFC). Dr. Rajji will describe the results of a study which assessed deficits in coupling in response to a brain stimulation intervention delivered to the DLPFC in healthy people and people with schizophrenia, using a paired associative stimulation (PAS) paradigm. He will show that people with schizophrenia are impaired compared to the healthy subjects who also received PAS on PAS-induced potentiation of cortical evoked activity or coupling, compared with a PAS control intervention. Dr. Rajji will then describe how PAS paradigms can be used to improve brain plasticity and working memory.

Third, Dr. Aristotle Voineskos will describe a novel diffusion tensor imaging (DTI)-based approach that can index biomarkers of normal and pathological aging. While DTI studies have shed light on white matter microstructural abnormalities associated with aging, the focus has been on deep white matter (DWM) tracts, the long-range connections between brain regions. We now have a method to measure superficial white matter (SWM), which can index local brain connectivity, and may be important for cognitive function. Dr. Voineskos will describe results from a large study of normal aging individuals across the adult lifespan who underwent DTI and a full cognitive battery. Areas of SWM susceptible to aging and areas predicting cognitive 
performance will be described. The results of this study show that SWM declines in integrity with age, and could be a key neural substrate for age-related cognitive decline. SWM should be considered as an early outcome biomarker in clinical intervention studies aimed at improving or preserving cognitive performance in late-life.

Finally, Dr. Benoit Mulsant, the symposium discussant, will provide perspective regarding biomarker utilization in clinical trials and interventions, particularly in light of new position statements from major funding agencies expecting the use of biomarkers in such studies.

\section{Transcranial Magnetic Stimulation Neurophysiology in Late-Life Depression}

Daniel M. Blumberger, MD, MSc, FRCPC

Late-Life Mood Disorders Clinic, Centre for Addiction and Mental Health

\section{Learning Objectives:}

1. To learn about transcranial magnetic stimulation (TMS) as a neurophysiological probe.

2. To understand previous TMS neurophysiology findings in depression.

3. To appreciate preliminary findings from a prospective study of TMS neurophysiology in the context of a latelife depression treatment trial.

Background: Transcranial magnetic stimulation (TMS) is a non-invasive form of brain stimulation that capitalizes on the ability of time-varying magnetic fields to induce electrical currents in biological tissue via the principle of electromagnetic induction. TMS can provide an index of GABA receptor-mediated inhibition and glutamatergicmediated neurotransmission because it differentially stimulates inhibitory interneurons and pyramidal neurons in the motor cortex. There are several TMS paradigms that provide a measure of GABA receptor-mediated inhibitory neurotransmission: long interval cortical inhibition (LICI), the cortical silent period (CSP), and short-interval cortical inhibition (SICI). Glutamatergic neurotransmission can be assessed using a paradigm called intracortical facilitation (ICF) and long-term potentiation (LTP)-like neuroplasticity can be assessed using a paradigm called paired associative stimulation (PAS). Dysfunctional cortical inhibition (CI) and neuroplasticity have been postulated as a neurophysiological mechanism involved in treatment resistant depression (TRD). These paradigms also have potential to predict response to existing psychopharmacological treatments. Younger adults with depression demonstrate deficits in SICI and CSP and SICI deficits are specifically associated with treatment resistant depression. Aging is also associated with alterations in $\mathrm{CI}$.
Methods: We examined cortical inhibition and neuroplasticity TMS paradigms in 52 older adults with depression. SICI, ICF, CSP, and PAS were measured prior to treatment with Venlafaxine in 52 depressed older adults and again after 12 weeks of treatment. A group of age and gender matched controls were tested and recruited on a 2-to-1 basis.

Results: Data will be presented from three groups: non-depressed older adults, depressed older adults without treatment resistance, and depressed older adults with treatment resistance. In addition, neurophysiological predictors of response to treatment with Venlafaxine will be presented.

Conclusions: TMS neurophysiology paradigms hold potential as biological predictors of treatment response and markers of illness severity.

\section{Deficits in Neuroplasticity and Theta-Gamma Coupling in Patients with Schizophrenia}

Tarek K. Rajji, $\mathrm{MD}^{1,2}$, Reza Zomorrodi-Moghaddam, $\mathrm{PhD}^{1,2}$, Lisa Tran, BA $^{1,2}$, Bahar Salavati, MS $^{1,2}$, Rae Price, BS $^{1,2}$, Daniel M. Blumberger, MD, $\mathrm{MS}^{1,2}$, Benoit H. Mulsant, ${ }^{1}$, $\mathrm{MS}^{1}$, Zafiris J. Daskalakis, $\mathrm{MD}, \mathrm{PhD}^{1,2}$

${ }^{1}$ Campbell Family Mental Health Research Institute at the Centre for Addiction and Mental Health, and University of Toronto; ${ }^{2}$ Temerty Centre for Therapeutic Brain Intervention at the Centre for Addiction and Mental Health

\section{Learning Objectives:}

1. Learn about a method to assess neuroplasticity in vivo using brain stimulation.

2. Learn about deficits in neuroplasticity in patients with schizophrenia.

3. Learn about a novel marker of working memory: thetagamma coupling.

Background: Deficits in working memory are core features in schizophrenia and depend on functional dorsolateral prefrontal cortex (DLPFC). Modulation of gamma oscillations amplitude by theta oscillations phase ("theta-gamma coupling") is thought to mediate working memory. This study assessed deficits in coupling in response to a brain stimulation intervention delivered to the DLPFC.

Methods: Paired Associative Stimulation (PAS), combined with electroencephalography (EEG) was applied to the DLPFC of 25 healthy subjects (mean age $=36.2, \mathrm{SD}=16.1$ ) and 22 subjects with schizophrenia (mean age $=36.3$, SD $=12.0)$. PAS is a transcranial magnetic stimulation based intervention that induces long-term potentiation-like increase in cortical evoked activity (CEA) and coupling. A PAS control intervention (PAS-C) was administered 
to a third group of 15 healthy subjects (mean age $=32.9$, $\mathrm{SD}=11.6)$.

Results: Compared with those who received PAS-C, the healthy subjects who received PAS experienced significant potentiation of CEA and coupling as captured over the DLPFC (all $p<.005)$. Subjects with schizophrenia also experienced significant potentiation of CEA and coupling in response to PAS compared to baseline or those who received PAS-C (all $p<.005$ ). However, they were impaired compared to the healthy subjects who received also PAS on PAS-induced potentiation of CEA or coupling (all $p<.001)$.

Conclusions: Patients with schizophrenia experience deficits in PAS-induced potentiation of cortical activity and thetagamma coupling in the DLPFC. These findings suggest that disrupted plasticity in DLPFC networks translates into abnormal theta-gamma coupling and consequently working memory deficits in patients with schizophrenia.

\section{A Novel White Matter Biomarker of Cognitive Performance Across the Lifespan}

Aristotle Voineskos, MD, $\mathrm{PhD}, \mathrm{FRCPC}^{1}$, Arash Nazeri, $\mathrm{MD}^{2}$

${ }^{1}$ Kimel Family Translational Imaging-Genetics Laboratory, Centre for Addiction and Mental Health; ${ }^{2}$ Centre for Addiction and Mental Health

\section{Learning Objectives:}

1. To learn about diffusion-based MRI of white matter as a tool to identify brain-based biomarkers.

2. To understand previous diffusion-based MRI findings of white matter and how they relate to cognitive performance in healthy and pathological aging.

3. To understand why changes in superficial white matter may be a useful biomarker of clinical response.

Background: Normal aging is accompanied by continuous decline in cognitive abilities. In the recent years, diffusion tensor imaging (DTI) studies have shed light on white matter microstructural abnormalities associated with aging. However, these studies have focused on deep white matter (DWM) tracts, the long-range connections between brain regions. We now have a method to measure superficial white matter (SWM), which connects the gray matter to the white matter and serves to ensure local brain connectivity. The purpose of the present study was to determine whether superficial white matter measures can serve as biomarkers of cognitive performance.

Methods: 141 healthy individuals (F/M: 74/67) across the adult lifespan (18-85 years) underwent DTI and performed a battery of cognitive tasks. Cognitive/behavioural tests were submitted to a principal component analysis. Following image analysis, the relative contribution of SWM and DWM to age-related cognitive decline was assessed using multivariate modelling.

Results: Significant linear age-related decline of fractional anisotropy (FA) values was observed in much of SWM. SWM-FA adjacent to both precentral gyri and right frontal cortex predicted fine motor speed, while performance in attention/visuomotor coordination correlated with SWM-FA in the frontal left hemisphere, along with right parieto-occipital SWM-FA ( $p$ value $<.05)$.

Conclusions: Our results show that SWM declines in integrity with age, and could be a key neural substrate for age-related cognitive decline. SWM FA should be considered as an early outcome biomarker in clinical intervention studies aimed at improving cognitive performance in late life.

Telepsychiatry: Clinical and

S2 Educational Applications in Geriatric Psychiatry

Cindy Grief, MD, FRCPC, MSc ${ }^{1}$, Lisa Sokoloff, MS, CCC-SLP, Reg. $\mathrm{CASLPO}^{2}$, David Conn, MB, FRCPC ${ }^{3}$, Rob Madan, MD, FRCPC 4 ${ }^{1}$ Department of Psychiatry, Baycrest; ${ }^{2}$ International Relations and Interprofessional Education, Baycrest; ${ }^{3}$ Centre for Education and Knowledge Exchange in Aging, Baycrest; ${ }^{4}$ Centre for Mental Health, Geriatric Psychiatry, Baycrest

\section{Learning Objectives:}

1. Describe the many uses of videoconferencing in geriatric psychiatry.

2. Identify the benefits and limitations to using videoconferencing for clinical and educational purposes.

3. List ways for utilizing videoconferencing to enhance clinical care and education in your respective practice.

Background: Telepsychiatry is a cost and time-efficient means for improving services to under-resourced sectors including geriatric mental health. At Baycrest, several initiatives are underway to increase service provision to older adults across the province of Ontario using videoconferencing technology.

Methods: In the following presentations we highlight the relevance and potential applicability of videoconferencing in clinical care and education in geriatric mental health.

Results: We have implemented a national videoconference education series with a linked online Community of Practice, a project that involves the collaboration of family physicians and other allied health-care professionals. We also offer telepsychiatry consultations to Northern Ontario, and are actively involved in training psychiatry residents at the University of Toronto. 
Conclusions: This symposium will share our successes and challenges in using telepsychiatry, and outline ways to incorporate videoconferencing technology into practice.

\section{Incorporating Telepsychiatry and Distance Education into Core Training in Geriatric Psychiatry}

David Conn, MB, $\mathrm{FRCPC}^{1}$, Cindy Grief, $\mathrm{MD}$, FRCPC, $\mathrm{MSc}^{2}$, Rob Madan, MD, FRCPC ${ }^{3}$

${ }^{1}$ Centre for Education and Knowledge Exchange in Aging, Baycrest; ${ }^{2}$ Department of Psychiatry, Baycrest; ${ }^{3}$ Centre for Mental Health, Geriatric Psychiatry, Baycrest

\section{Learning Objectives:}

1. Discuss the benefits of telepsychiatry and videoconferencing technology in medical education.

2. Describe the use of telepsychiatry in training residents in geriatric psychiatry.

3. Discuss ways to further incorporate telepsychiatry and videoconferencing into residency training in geriatric psychiatry.

Background: Current literature supports the use of distance technology in education, both for group learning and clinical skills development. The scope of potential uses for videoconferencing technology for education is growing rapidly. At the University of Toronto, resident training in telepsychiatry is an emerging priority highlighted in the Department of Psychiatry's strategic plan. Baycrest is a fully-affiliated teaching hospital in Toronto that offers PGY3 residents core training in geriatric psychiatry. Beyond participating in videoconferenced education rounds, residents at our centre are invited to provide telehealth consultations to underserviced communities and collaborate with health-care teams in remote communities across Northern Ontario. The role of videoconferencing and telepsychiatry in the education of resident trainees in geriatric psychiatry is examined.

Methods: The literature on training in telepsychiatry will be reviewed with a focus on learning objectives. Informal feedback was obtained from resident trainees participating in weekly geriatric mental health assessments to sites in Northwestern Ontario. Additionally, reflections on the process of supervising residents for a telepsychiatry rotation are captured.

Results: Health-care teams and client recipients of telepsychiatry services report high satisfaction rates with care. Clinical experiences are also highly rated by resident trainees in geriatric psychiatry.

Conclusions: Participating in telehealth consultations in geriatric mental health within geographically remote communities impacts positively on trainees' education in geriatric psychiatry. Benefits of can be mapped according to CANMEDS competencies. Ideas for further enhancing the educational experience of resident trainees are highlighted.

\section{Enhancing the Care of Older Adults Through Telepsychiatry}

David Conn, MB, FRCPC ${ }^{1}$, Rob Madan, MD, FRCPC ${ }^{2}$

${ }^{1}$ Centre for Education and Knowledge Exchange in Aging, Baycrest; ${ }^{2}$ Centre for Mental Health, Geriatric Psychiatry, Baycrest

\section{Learning Objectives:}

1. Describe how a University-affiliated geriatric telepsychiatry service collaborates with a rural psychogeriatric outreach team.

2. Discuss the benefits and challenges of running a geriatric telepsychiatry program.

3. Understand options for program evaluation of a clinical service.

Background: Weekly telepsychiatry consultations have been provided since 2002 to six communities in Northwest Ontario. Staff from a single community psychogeriatric outreach service working within these communities facilitate the referrals.

Methods: Program evaluation included a chart review, patient and staff evaluations, a survey administered to all physicians in referring communities, and focus groups of staff working in local community agencies.

Results: The mean age at the time of consultation was 76.7 years. Sixty-eight per cent of patients were females. The most frequent diagnoses were dementia (54\%), depression (28\%), and mild cognitive impairment (19\%). The most frequent medication recommendations were antidepressants or cholinesterase inhibitors. Two hundred ninety-four patient assessments and case consultations were carried out over an 8-year period. Post-session evaluation surveys rated the provision of information, whether objectives were met, and overall usefulness of recommendations. The mean scores were between $4.6-4.85$ on a 5 -point scale. Referring physicians were confident and satisfied with the recommendations made. All planned to continue using telepsychiatry as a future career option. Focus groups added useful information about challenges and potential barriers to utilizing the program.

Conclusions: The program was rated as being highly valued across all modalities of evaluation. Members of the referring team believe that access to a geriatric psychiatrist has broadened the team's knowledge base and its use of assessment tools, and increased their ability to better construct their patients' treatment plans. 


\section{Linking Videoconferencing with an Online Community of Practice}

Cindy Grief, MD, FRCPC, MSc ${ }^{1}$, Lisa Sokoloff, MS, CCC-SLP, Reg. CASLPO $^{2}$, David Conn, MB, FRCPC ${ }^{3}$, Rob Madan, MD, FRCPC ${ }^{4}$ ${ }^{1}$ Department of Psychiatry, Baycrest; ${ }^{2}$ International Relations and Interprofessional Education, Baycrest; ${ }^{3}$ Centre for Education and Knowledge Exchange in Aging, Baycrest; ${ }^{4}$ Centre for Mental Health, Geriatric Psychiatry, Baycrest

\section{Learning Objectives:}

1. Describe the development of a national videoconference education series in geriatric mental health.

2. Discuss the challenges of developing an online community of practice linked with this videoconference series.

3. Identify ways to optimize interactivity and engagement of participants during live and virtual exchanges.

Background: A previous needs assessment distributed to health-care professionals indicated interest in ongoing videoconferenced education sessions in topics pertaining to geriatric mental health. A monthly videoconferenced education series was developed and has been linked with an online community of practice (CoP). This CoP offers a virtual space for health-care professionals to connect pre- and postlive videoconferenced sessions and is intended to provide a framework for ongoing discussion and knowledge exchange.

Methods: Needs assessment surveys were developed and disseminated nationally to health professionals interested in geriatric mental health including underserviced populations and family physicians. Results were used to inform the format and content of the education sessions and development of the CoP. A database of partners was generated from national organizations, professional, and administrative contacts.

Results: $51 \%$ somewhat comfortable, $41 \%$ very comfortable, and only $8 \%$ not comfortable at all with participation in live broadcasts $(N=43)$. Currently, monthly broadcasts average more than 15 connecting sites and membership in the CoP has exceeded 100. Videoconference sessions have expanded to include simultaneous and archived webcasting.

Conclusions: Interactivity during live sessions varies with presenter. Guidelines for speakers are being developed. Means for enhancing engagement in online forum being actively explored and include securing Mainpro credits for family physicians.

Thinking Critically about

S3 Dementia through Theatre and Arts based Practices

Aynsley Moorhouse, MA, MFA, MSW ${ }^{1}$, LJ Nelles, MFA, $\mathrm{PhD}^{2}$, Jenny Carr, OT Reg. (Ont.) ${ }^{2,3}$
${ }^{1}$ Dotsa Bitove Wellness Academy, UHN; ${ }^{2}$ Cyril \& Dorothy, Joel \& Jill Reitman Centre for Alzheimer's Support and Training, Mount Sinai Hospital; ${ }^{3}$ Department of Psychiatry, Mount Sinai Hospital

\section{Learning Objectives:}

1. Participants will become familiar with different applications of theatre to health care to observe and understand complexities in patient narrative.

2. Participants will understand the potential for theatre in to enhance personhood and relationship.

3. Participants will discuss possible interventions/applications to their work in geriatric psychiatry.

Background: The malleable nature of theatre results in multiple intersections with health care, including therapeutic practice (theatre-based research), data dissemination or knowledge translation (research-based theatre), and increased self-awareness and understanding of patient narrative. Ethnographer Dwight Conquergood ${ }^{(1)}$ promoted the "epistemological potential of performance as a way of deeply sensing the other" (p. 3). This symposium looks at multiple ways that theatre can be used to understand the lived experience of dementia. We examine how engagement in artistic practice can help to re-imagine narratives and enhance quality of life.

Methods: This symposium presents the work of three artsin-health practitioners working with dementia. We will demonstrate the potential of theatre to inform, reflect, and enhance relationship. We will look at the process of creating a performance-based media art project with a person with dementia, the development of a theatrical collage based on the lived experience of simulating dementia, and direct intervention that uses theatre (and other arts practices) to create meaningful expressive and social experiences for people with dementia and carers. We will address the problems inherent in interdisciplinary work and look at how dissemination of this work has created important dialogue about dementia and personhood.

Results: Theatre can be a tool for research, education, and intervention. It provides an opportunity for health-care practitioners to understand complex patient narratives, promote discussion between families and health practitioners, and enrich and enhance the lives of families experiencing dementia. Conclusions: The thoughtful use of arts based practice to explore and understand the complexity of dementia has potential in health care.

1. Conquergood D. Performing as a moral act: ethical dimensions of the ethnography of performance. Literature in Performance. 1985;2(5):1-13. 


\section{Using theatre and Arts-based Programming to Promote Mental Health with People Who Have Dementia}

\author{
Jenny Carr, OT Reg. (Ont.) ${ }^{1,2}$, LJ Nelles, MFA, PhD ${ }^{1}$, Aynsley Moor- \\ house, MA, MFA, MSW ${ }^{3}$ \\ ${ }^{1}$ Cyril \& Dorothy, Joel \& Jill Reitman Centre for Alzheimer's \\ Support and Training, Mount Sinai Hospital; ${ }^{2}$ Department of \\ Psychiatry, Mount Sinai Hospital; ${ }^{3}$ Dotsa Bitove Wellness \\ Academy, UHN
}

\section{Learning Objectives:}

1. Attendees will be become familiar with aspects of mental health that may be possible to impact through engaging in arts-based group activities.

2. Attendees will learn about an approach to theatre and arts-based programming to promote mental health for people with dementia.

3. Attendees will learn about potential benefits to carer-care-recipient dyads who incorporate the arts into their relationship.

Background: The Reitman Centre Carers Program offers a concurrent arts-based program for family members with dementia (FWD) as part of a therapeutic intervention for family caregivers. There is evidence that interventions to decrease burden for carers have more success when parallel programming is available for the FWD. ${ }^{(1)}$ Arts-based activities, such as theatre, are thought to have a positive effect on quality of life and certain symptoms of dementia. For instance, theatre may improve communication, focus and a sense of well-being in FWD. ${ }^{(2)}$

Methods: Small-group arts activities prompt social interaction and engagement in meaningful activities. To promote health in the broadest sense, a different art modality is used each week. Theatre-based activities have included drama games, play reading, and improvisation. Carers are informed about the activities that have taken place. A positive relationship between carer and family member for whom they provide care has also been shown to decrease burden.

Results: Participants are generally engaged in the activities and discussions. In order to transfer any positive effects of the group to the home environment a study has been completed that asks carers what they would like to know about family members' engagement in the groups. An effective feedback process to carers is in development.

Conclusions: Non-pharmacological interventions, such as theatre and the arts, can offer opportunities for addressing mental health by facilitating social contact and engagement in meaningful activities.(2) Arts-based programming is a valuable tool to approach health-care intervention for both members of a family dyad in the context of dementia.
1. Schulz R, Martire LM, Klinger JN. Evidence-based caregiver interventions in geriatric psychiatry. Psychiatry Clin N Am. 2005;28(4):1007-38.

2. Phillips LJ, Reid-Arndt SA, Pak Y. Effects of a creative expression intervention on emotions, communication, and quality of life in persons with dementia. Nurs Res. 2010;59(6):417-25.

\section{Rewriting Dementia Through Media Art}

Aynsley Moorhouse, MA, MFA, MSW ${ }^{1}$, LJ Nelles, MFA, PhD ${ }^{2}$, Jenny Carr, oT Reg. (Ont.) $)^{2,3}$

${ }^{1}$ Dotsa Bitove Wellness Academy, UHN; ${ }^{2}$ Cyril \& Dorothy, Joel \& Jill Reitman Centre for Alzheimer's Support and Training, Mount Sinai Hospital; ${ }^{3}$ Department of Psychiatry, Mount Sinai Hospital

\section{Learning Objectives:}

1. Attendees will learn about the potential of arts interventions to affect multiple levels of function with respect to personhood, engagement, and interpersonal relationships.

2. Attendees will learn how arts-based initiatives can used as a powerful tool to challenge pervasive negative dementia discourses.

3. Attendees will learn how to use narrative-based arts interventions within their clinical practice to improve clients' quality of life and well-being.

Background: With dementia on the rise, it is imperative that we rethink our beliefs about selfhood. The current negative discourse surrounding dementia is exceedingly harmful for PWD, family members, and health professionals alike, and has led to a culture of hopelessness even within the health-care context. When my father was diagnosed with Alzheimer's disease, I challenged this pervasive discourse through my artistic practice, and chose to examine personhood by rewriting the dementia narrative. Instead of working through this alone, my father and I worked together.

Methods: Over a 3-year period, I recorded eight hours of conversations between my father and me. Using these recordings, I created a series of audio installations that represent my father's life, his experience with dementia and, perhaps most importantly, our relationship.

Results: This talk will describe how the project has given my father voice, has enhanced our relationship, and has taught me about personhood in the face of memory loss. In addition, I will outline how the artwork has become a powerful knowledge translation tool, where my father teaches audience members about his experience with dementia. The pieces are intimate, immersive, personal and raw, and they elicit a strong emotional response from audience members. 
Conclusions: Arts-based initiatives are a powerful personcentered approach to dementia care. They have the potential to improve quality of life by affecting multiple levels of function with respect to personhood, active engagement and relationship building. In addition, they can challenge pervasive negative dementia discourses.

\section{Understanding Dementia Through Theatre}

LJ Nelles, MFA, PhD ${ }^{1}$, Aynsley Moorhouse, MA, MFA, MSW ${ }^{2}$, Jenny Carr, OT Reg. (Ont.) ${ }^{2,3}$

${ }^{1}$ Cyril \& Dorothy, Joel \& Jill Reitman Centre for Alzheimer's Support and Training, Mount Sinai Hospital; ${ }^{2}$ Dotsa Bitove Wellness Academy, UHN; ${ }^{3}$ Department of Psychiatry, Mount Sinai Hospital

\section{Learning Objectives:}

1. Participants will be introduced to concepts and examples from performance theory as a foundation for understanding how using theatre can promote patient centred care, research dissemination, and dialogue.

2. Participants will examine the potential for theatre or aesthetic intervention as a means of understanding the complexities of the lived experience of illness.

3. Participants will reflect and discuss possible strategies for translation /implementation in their programs.

Background: Intersections of theatre and health provide innovative opportunities to enhance self-awareness, critical reflection, and narrative understanding. Evidence from performance studies and cognitive neuroscience suggest embodied processes inherent to performance can enhance empathy and self-awareness. This talk describes a play created by artist-standardized patients with experience in the simulation of dementia. Our task was to investigate the stories and perspectives of dementia that had been written on our bodies through interactions with families, students, and clinicians. Our goal was to capture, through performance, the multiple perspectives and complexities faced by those whose lives are touched by dementia.

Methods: The creative research process will be examined along with other methods of applying theatre to research for data dissemination and knowledge translation. Our process applied qualitative descriptive methodology to written and physical exploration to determine themes. From the themes came the narrative and gestural language. Live music personified the clinical aspects of the illness. The result was a post-modern theatrical collage of broken narratives.

Results: The stories are not those of individuals; rather they are an amalgam of elements from hundreds of stories that borrowed our bodies, universal stories of illness experience, stories that express our relationship with the human experience of dementia. Multiple performances were well received and resulted in dynamic discussions with practitioners, patients, and family members.

Conclusions: A theatrical frame can result in both a critical and visceral understanding of the lived experience of disease that can influence the practitioner-patient relationship.

Brain Stimulation

S4 Interventions in Late Life

Mood and Cognitive

Wednesday

2:15 p.m.

Disorders

Sanjeev Kumar, MD $^{1}$, Tarek K. Rajji, MD, FRCPC ${ }^{2}$, Daniel M. Blumberger, MD, MSc, FRCPC $^{3}$

${ }^{1} \mathrm{CAMH} ;{ }^{2}$ Division of Geriatric Psychiatry, CAMH; ${ }^{3}$ LateLife Mood Disorders Clinic, CAMH

\section{Learning Objectives:}

1. To learn about importance of preventive strategies for Alzheimer's disease (AD) and related disorders and discuss study design of a multi-site preventative study using cognitive remediation and trancranial direct current stimulation.

2. To learn about potential role of TMS-EEG in diagnosis and management of $\mathrm{AD}$ and appreciate preliminary findings from a study using paired associative stimulation.

3. To learn about role of rTMS in late life depression and appreciate preliminary findings from a study of deep rTMS using a novel coil configuration.

Background: Late-life depression (LLD) and Alzheimer's disease (AD) pose a major health challenge in ageing population across the globe. Neurophysiology of these disorders is poorly understood and there is a relative lack of effective treatments. Up to $84 \%$ of individuals treated for LLD either do not respond to treatment or respond and then relapse. They are more likely to develop dementia even after successful treatment of depression. Similarly, currently available medications for dementia are not very efficacious. Electrical and magnetic brain stimulation interventions such as transcranial magnetic stimulation (TMS) and transcranial direct current stimulation (tDCS) are increasingly being studied, and there is an urgent need to further develop and refine the use these interventions in elderly.

Methods: We present: 1) study design of a randomized controlled trial using cognitive remediation combined with tDCS $(\mathrm{CR}+\mathrm{tDCS})$ to prevent the conversion of cognitively normal LLD subjects to MCI or dementia or the conversion of those with MCI to dementia; 2) pilot data from a TMS EEG study, evaluating working memory deficits and neuroplasticity in early $\mathrm{AD}$ using paired associative stimulation; 3) blinded data 
from an ongoing study of deep rTMS in LLD. Feasibility of recruitment, tolerability, protocol adherence, and efficacy will be discussed.

Conclusions: Brain stimulation interventions are poised to play increasingly significant role in late life mood and cognitive disorders. 1) $\mathrm{CR}+\mathrm{tDCS}$ may have the potential for universal prevention or for selective prevention of $\mathrm{AD}$ and related disorders. 2) TMS-EEG may help develop novel biomarkers of neuroplasticity and impaired working memory in early AD. 3) Deep rTMS may be an effective option for treatment resistance in LLD.

\section{Deep Repetitive Transcranial Magnetic Stimulation in Late-life Depression}

Daniel M. Blumberger, MD, MSc, FRCPC ${ }^{1}$, Tarek K. Rajji, MD, FRCPC $^{2}$, Benoit Mulsant, MD, MS, FRCPC ${ }^{3}$, Z. Jeff Daskalakis, $\mathrm{MD}, \mathrm{PhD}, \mathrm{FRCPC}^{3}$

${ }^{1}$ Late-Life Mood Disorders Clinic, CAMH; ${ }^{2}$ Division of Geriatric Psychiatry, CAMH; ${ }^{3} \mathrm{CAMH}$

\section{Learning Objectives:}

1. To learn about repetitive transcranial magnetic stimulation (rTMS) as a treatment for late-life depression.

2. To review previous rTMS treatment studies which have included older adults and understand the potential benefits of deep rTMS.

3. To appreciate preliminary findings from a study of deep rTMS using a novel coil configuration.

Background: Up to $84 \%$ of individuals treated for late-life depression (LLD) either do not respond to treatment or respond and then relapse. Strategies for managing treatment resistance in older adults are problematic due to increased sensitivity to side effects and co-morbid medical illnesses. Repetitive transcranial magnetic stimulation (rTMS) involves stimulating the brain with a series of magnetic field pulses. rTMS has been found to be an effective treatment in younger and middle-aged adults who have failed a moderate number of treatments. However, few studies have evaluated rTMS for older patients with LLD. There is some limited evidence of efficacy for rTMS in older adults; several factors have limited the overall efficacy of this treatment in older adults. The major factors responsible for limited efficacy found in previous studies are changes in the brain that occur with normal aging and the reduced size of the magnetic field generated by conventional coils. Deep rTMS may be able to achieve improved outcomes due to the size of the magnetic field generated by this coil.

Methods: Patients over the age of 60 who had failed at least one adequate medication trial are being randomized to either active treatment or placebo stimulation. Treatment is administered daily, five days per week, for between 30 and 45 minutes for four weeks. The treatment intensity is set at $120 \%$ motor threshold.

Results: Blinded data will be presented from the ongoing study. Feasibility of recruitment, tolerability, protocol adherence, and efficacy data will be presented.

Conclusions: Deep rTMS may be an effective option for treatment resistance in LLD.

Dorsolateral Prefrontal Cortex Neuroplasticity and Working Memory Function in Patients with Early Alzheimer's Disease: Preliminary Results from a TMS-EEG Study

Sanjeev Kumar, $\mathrm{MD}^{1}$, Reza Zomorrodi-Moghaddam, $\mathrm{PhD}^{1}$, Rae Price, Bs $^{1}$, Daniel M. Blumberger, MD, MSc, FRCPC ${ }^{2}$, Tiffany Chow, MD, FRCPC ${ }^{1}$, Aristotle Voineskos, MD, PhD, FRCPC ${ }^{3}$, Corrinne Fischer, MD, FRCPC ${ }^{4}$, Bruce G. Pollock, MD, PhD, FRCPC ${ }^{1}$, Benoit Mulsant, MD, MS, FRCPC ${ }^{1}$, Z. Jeff Daskalakis, MD, PhD, FRCPC $^{1}$, Tarek K. Rajji, MD, FRCPC ${ }^{5}$

${ }^{1} \mathrm{CAMH} ;{ }^{2}$ Late-Life Mood Disorders Clinic, CAMH; ${ }^{3} \mathrm{Kimel}$ Family Translational Imaging-Genetics Laboratory, CAMH; ${ }^{4}$ Geriatric Psychiatry, Saint Michael's Hospital; ${ }^{5}$ Division of Geriatric Psychiatry, CAMH

\section{Learning Objectives:}

1. To learn about neuroplasticity as measured by potentiation of cortical evoked activity and theta-gamma coupling.

2. To learn about relevance of theta-gamma coupling and potentiation of cortical evoked activity in Alzheimer's disease.

3. To learn about potential role of TMS-EEG in diagnosis and management of Alzheimer's disease.

Background: Deficits in frontal lobe functions including deficits in working memory are common across all stages of Alzheimer's disease (AD). Working memory is related to frontal cortical oscillations, particularly theta-gamma coupling. Theta-gamma coupling depends on robust synaptic neuroplasticity. Paired associative stimulation (PAS) is a brain stimulation approach that assesses neuroplasticity in the frontal cortex when combined with electroencephalography (PAS-EEG). In this study, we are using PAS-EEG to assess, for the first time, neuroplasticity in the dorsolateral prefrontal cortex (DLPFC) of patients with AD. We are also assessing these patients' performance on a working memory task and associated theta-gamma coupling in the frontal cortex.

Methods: Seven participants with early AD and seven healthy participants were administered the N-back task while EEG was being recorded to assess working memory and associated theta-gamma coupling in the frontal cortex. Then, they were administered PAS-EEG to assess neuroplasticity in the DLPFC. 
Results: Participants with AD performed worse on the Nback task (Cohen's $\mathrm{d}=1.46, p=.02$ ) than healthy participants. They also demonstrated deficits in theta-gamma coupling during N-back performance when compared to healthy participants (Cohen's $d=1.56, p=.03$ ). Finally, they demonstrated a trend towards impaired DLPFC neuroplasticity as assessed by PAS-EEG (Cohen's $d=0.97$ ).

Conclusions: These preliminary results from an ongoing study suggest that impaired working memory in patients with $\mathrm{AD}$ is mediated by impaired theta-gamma coupling and that these deficits are related to impaired frontal lobe neuroplasticity. Our findings need to be extended and confirmed in a larger sample size.

Prevention of Alzheimer's Dementia in High-risk Populations: a Randomized Controlled Trial of a Combination of Cognitive Remediation and Brain Stimulation: Study Design

Tarek K. Rajji, MD, FRCPC ${ }^{1}$, Christopher R. Bowie, $\mathrm{PhD}^{1}$, Daniel M. Blumberger, MD, MSc, FRCPC $^{2}$, Benoit Mulsant, MD, MS, FRCPC ${ }^{1}$ ${ }^{1} \mathrm{CAMH} ;{ }^{2}$ Late-Life Mood Disorders Clinic, CAMH

\section{Learning Objectives:}

1. To learn about rational behind using transcranial direct current stimulation and cognitive remediation for prevention of Alzheimer's disease.

2. Learn about the feasibility of the study.

3. Learn about the study design, hypothesis, and protocol.

Background: Mild cognitive impairment (MCI) is considered a prodromal condition for dementia. Late-life depression (LLD) has been identified as one of the most promising targets for $\mathrm{AD}$ prevention studies. We propose to study the efficacy of a preventive intervention for $\mathrm{AD}$ in three high-risk groups: MCI, LLD without MCI, and LLD with MCI. Proposed intervention is a combination of cognitive remediation (CR) and non-invasive brain stimulation - transcranial direct current stimulation (tDCS).

Hypotheses: Over 24 months, five Toronto academic sites will enroll: 1) 125 non-depressed subjects age 60 and older who present with MCI; 2) 125 non-demented subjects age 65 and older who present without MCI but with an episode of major depression that has responded to antidepressant treatment; and 3) 125 non-demented subjects age 65 and older who present with MCI and an episode of major depression that has responded to antidepressant treatment These 375 subjects will be randomized under double-blind conditions to receive either $\mathrm{CR}+\mathrm{tDCS}$ or sham-CR + sham-tDCS and followed for 30 to 59 months.

Results: The rationale and design of the study protocol will be presented.
Conclusions: If successful, CR $+\mathrm{tDCS}$ can be tested in, and extended to, the general population or other populations at high risk for $\mathrm{AD}$.

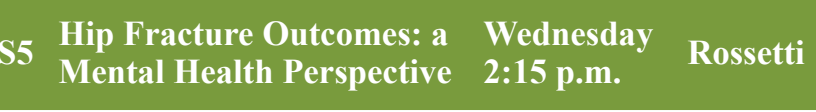

Our symposium will focus on mental health issues which impact on outcomes after hip fracture. Dr. Seitz will present his epidemiological research on the impact of dementia in outcomes for older adults with hip fractures. Dr. McGilton will discuss the implementation of models of care for supporting older adults with dementia in rehabilitation. Dr. Iaboni will discuss mood disturbance and other psychiatric symptoms after hip fracture, and will present data about the initiation of antidepressants and other psychotropic medications after hip fracture. The symposium will conclude with a panel discussion about optimizing care for older adults after hip fracture, from the perspective of mental health.

\section{Antidepressants and Other Psychotropic Medications After Hip Fracture}

Andrea Iaboni, MD, DPhil ${ }^{1}$, Dallas Seitz, $\mathrm{MD}^{2}$, Kathy McGilton, $\mathrm{RN}, \mathrm{PhD}^{3}$

${ }^{1}$ University of Toronto; ${ }^{2}$ Queen's University; ${ }^{3}$ Toronto Rehabilitation Institute-UHN

\section{Learning Objectives:}

1. To review the relationship between hip fracture and depression.

2. To characterize the use of antidepressant and other psychotropic medication after hip fracture.

3. To consider potential benefits and hazards of psychotropic use after hip fracture.

Background: Depression is common in older adults after hip fracture. The benefits and safety of antidepressants post-hip fracture have not been established. Importantly, antidepressants have been linked to falls and fractures. Our aim was to describe antidepressant prescribing practices in communitydwelling older adults after hip fracture.

Methods: We conducted a retrospective cohort study using linked, population-based administrative data from Ontario for the period from April 2003 to March 2012. Our cohort consisted of seniors after hip fracture surgery who returned home from hospital or rehabilitation and who had not been exposed to an antidepressant in the year prior to their fracture $(N=25,438)$. We identified the 90 -day incidence of new antidepressant use in this population and compared new users of antidepressants to non-users. 
Results: The mean 90-day incidence of antidepressant initiation after hip fracture was $8.7 \%$. The most commonly initiated antidepressant was trazodone (39.3\%), followed by SSRIs $(36.8 \%)$. New antidepressant users were older than non-users $(p<.001)$, were on more medications at baseline $(p<.001)$, had higher rates of dementia $(p<.001)$, more previous falls $(p<.001)$, and were more likely to have had a rehabilitation admission $(p<.001)$. New users of antidepressants were at higher risk of exposure to atypical antipsychotic $(p<.001)$, typical antipsychotic $(p<.001)$, and benzodiazepine $(p<$ .001) medications in the 90 days at home after hip fracture compared to non-users.

Conclusions: The 90-day period after a hip fracture is associated with a high rate of initiation of antidepressants. The prescription of multiple psychotropic medications in this vulnerable population warrants further investigation.

Factors Influencing the Implementation of the PatientCentred Rehabilitation Model of Care for Older Adults with Cognitive Impairment

Kathy McGilton, $\mathrm{RN}, \mathrm{PhD}^{1}$, Dallas Seitz, $\mathrm{MD}^{2}$, Andrea Iaboni, MD, DPhil ${ }^{3}$

${ }^{1}$ Toronto Rehabilitation Institute-UHN; ${ }^{2}$ Queen's University; ${ }^{3}$ University of Toronto

\section{Learning Objectives:}

1. To understand factors influencing rehabilitation care for persons with cognitive impairment.

2. To understand strategies used during implementation of the model of care.

3. To understand the components of the Patient-Centred Rehabilitation Model of Care targeting persons with cognitive impairment.

Background: In 2011, Bone and Joint Decade Canada released a recommendation for accessible intensive structured rehabilitation programs for older persons with cognitive impairment (CI) (i.e., dementia or delirium). In response to this demand, the Patient-Centred Rehabilitation Model of Care targeting persons with CI (PCRM-CI) was developed. The PCRM-CI has now been empirically tested within three active rehabilitation units in Ontario, demonstrating positive outcomes for older adults' post-hip fracture. This presentation will highlight the key factors influencing the implementation of the model of care within the three rehabilitation sites.

Methods: At each of the three sites, focus groups and individuals interviews were conducted with staff $(n=23)$ and managers $(n=5)$ to determine key factors influencing the successful implementation of the PCRM-CI.
Results: In order to facilitate successful implementation of the PCRM-CI, important considerations should be made at each stage of the process. Prior to implementation, staff's concerns pre-implementation, and leaders' commitment and understanding of the model should be adequately addressed. During implementation, provisions should be made to address the need for ongoing education and training. Post-implementation, continued support should be provided to staff to ensure they are able to care for this vulnerable population of older adults.

Conclusions: New models of rehabilitation care targeting persons with CI living at home prior to the hip fracture can be implemented in active rehabilitation units. Improving care for this vulnerable population will include a change in the admission criteria and the delivery of care on active rehabilitation units. The PCRM-CI can be implemented in rehabilitation facilities with minimal financial burden. The Advance Practice Nurse plays a significant role in the implementation and sustainability of this model.

\section{Access to Rehabilitation and Postoperative Outcomes of Older Adults with Dementia who Sustain Hip Fractures}

Dallas Seitz, MD ${ }^{1}$, Andrea Iaboni, MD, DPhil ${ }^{2}$, Kathy McGilton, $\mathrm{RN}, \mathrm{PhD}^{3}$

${ }^{1}$ Queen's University; ${ }^{2}$ University of Toronto; ${ }^{3}$ Toronto Rehabilitation Institute, UHN

\section{Learning Objectives:}

1. To review relationship between dementia and hip fractures.

2. Examine access to rehabilitation for older adults with hip fractures in Ontario.

3. Understand the association between receipt of postoperative rehabilitation and outcomes for older adults with hip fractures and dementia.

Background: Older adults with dementia frequently experience hip fractures. Rehabilitation following hip fractures is important in order to reduce disability and functional decline. However, older adults with dementia may have restricted access to rehabilitation services following hip fracture. We evaluated the effects of access to postoperative rehabilitation and subsequent outcomes for older adult with dementia who experienced hip fractures.

Methods: We identified all older, community-dwelling adults with dementia who underwent surgery for hip fractures in the province of Ontario, Canada between 2003 and 2011. We categorized individuals into one of four possible post-fracture rehabilitation groups: in-patient rehabilitation (IPR); home care-based rehabilitation (HCR); complex continuing care $(\mathrm{CCC})$; or, no rehabilitation. The risks of 
admission to long-term care (LTC), post-fracture mortality, and post-fracture adverse events were then compared across rehabilitation groups after adjusting for potential confounders using multivariable regression.

Results: A total of 11,200 individuals with dementia experienced hip fractures during the study period. Of the total group, 4,494 (40.1\%) received no rehabilitation, 2,474 (22.1\%) were admitted to CCC, 1,157 (10.3\%) received HCR, and 3,075 (27.4\%) received IPR. As compared to no rehabilitation, IPR, $\mathrm{HCR}$, and $\mathrm{CCC}$ were each associated with a reduced risk of LTC admission within the first 2 years following discharge from hospital, and HCR was associated with the lowest risk of all the types of rehabilitation. When compared to no rehabilitation, all three forms of rehabilitation were associated with reduced risks of mortality, with the greatest effect observed with IPR (hazard ratio: 0.44, 95\% CI: 0.41-0.47).

Conclusions: Admission to post-fracture rehabilitation for older adults with dementia is associated with a reduced risk of LTC placement and mortality, although many individuals with dementia do not receive these services following hip fracture. Improving access to rehabilitation services for this vulnerable population may improve post-fracture outcomes and quality of life.

\section{PAPER ABSTRACTS}

Community Based Group

Intervention for Older Adults

1a. experiencing low mood

and stress: An Ontario

Pilot Project
Jennifer Hardy, Oт Reg. (Ont.) ${ }^{1}$, Scott Mitchell ${ }^{1}$, Nasreen Khatri, PhD, C.Psych ${ }^{2}$, Michaela Hynie, $\mathrm{PhD}^{3}$, Felicia Zhang, $\mathrm{BSc}^{4}$

${ }^{1}$ Ontario Division, Canadian Mental Health Association; ${ }^{2}$ Rotman Research Institute, Baycrest; ${ }^{3}$ York Institute for Health Research; ${ }^{4} \mathrm{CMHA}$ Ontario

\section{Learning Objectives:}

1. Participants will learn about a new, innovative, CBTbased group program to address older adults' mental health challenges, and how this program impacted older adults living in Ontario.

2. Participants will learn about the facilitators and barriers older adults face in accessing a community-based mental health program.

3. Participants will learn about initiating/carrying out a mental health-based pilot project, including the power of partnership.

Background: Living Life to the Full (LLTTF) is an 8-week, 12-hour course based on cognitive behavioural therapy (CBT) principles. LLTTF is offered in a group format and focuses on skill enhancement for those who want to maximize their ability to deal with life's challenges.

Methods: Canadian Mental Health Association (CMHA) branches across Ontario partnered with local Older Adult Centres' Association of Ontario (OACAO) members to offer LLTTF to participants aged 50 and up. Participants selfreferred to the program via community centre advertising. Registration was limited to 15 persons per course. Those enrolled were locally evaluated by peer research assistants. Measures of mood (BDI) and anxiety (BAI) were collected at baseline, post-course, and 3 months follow-up. Based on early results and results from a similar UK study using LLTTF, we hypothesized participants to experience a decrease in anxiety and depression.

Results: A total of 11 partnerships were built, with 63 older adults participating in the research evaluation across Ontario. Classes were well attended, with wait lists forming at various sites. From pre-intervention to post-intervention, participants demonstrated an overall improvement in mood, anxiety, and cognition scores. Participants reported that the top facilitators to attending the course were enjoyment of the facilitator, others in the group, as well as self-motivation to make a change. The top barriers to attending were scheduling, weather, and number of sessions.

Conclusions: LLTTF is effective in improving symptoms of depression and anxiety when delivered in a community setting. Community-based mental health approaches can be a successful model of mental health intervention when strategic partnerships are utilized.

$\begin{array}{lll}\text { 1b. Dementia and Staff Grief } & \text { Tuesday } \\ \quad \text { 10:30 a.m. } & \text { Scott }\end{array}$

Mary Schulz, MSw, Rsw

Alzheimer Society of Canada

\section{Learning Objectives:}

1. To understand how staff grief and loss impact their work with people with dementia in a variety of settings.

2. To hear examples of organization and peer-led strategies to support staff when a person dies.

3. To learn self-care techniques.

Background: Health-care providers working with people with dementia often experience a profound sense of grief when the person they support dies. In long-term care (LTC), it is estimated that up to $39 \%$ of residents are anticipated to die each year by the year 2020 (Fisher, Ross, McLean, 2000). The staff in these settings experiences the death of a client on a regular basis, yet few initiatives exist to address their grief and loss. Similarly in community-based settings, loss 
not only can result from client death, but also transition to other levels of care.

Methods: The Alzheimer Society of Canada (ASC) conducted thorough research reviews and Pan-Canadian interviews with health-care providers working with people with dementia where death is omnipresent.

Results: ASC developed a practical evidence-based resource outlining the feelings of loss and grief experienced by health-care providers working with people with dementia. The goal is to recognize, name, and address staff grief; change the culture of silence around death and dying; equip staff with practical strategies (self-care) to help them better support people with dementia throughout the progression of the disease until end of life; and to promote grief support among health-care organizations.

Conclusions: This guide provides recommendations on how organizations can offer support to staff, offers strategies for staff self-care, and includes practical case studies to address this complex issue.

Investigating the

Association between

2a. Perceived Autonomy and

Suicide Ideation among

Older Adults

Marnin Heisel, PhD, $\mathrm{CPsych}^{1}$, Gordon Flett, $\mathrm{PhD}^{2}$

${ }^{1}$ Department of Psychiatry, Western University; ${ }^{2}$ York University

\section{Learning Objectives:}

1. Participants will learn about the potential impact of selfexpectancies regarding need for autonomy and personal control on suicide risk in later life.

2. Participants will be able to discuss findings of a longitudinal study investigating the impact of perceived autonomy on the presence and/or development of suicide ideation among older adults.

3. Participants will engage in discussion of study implications regarding the potential identification of at-risk older adults and intervention to reduce risk for suicide.

Background: Older adults have a high suicide rate, necessitating models of psychological processes involved in the onset or exacerbation of suicide risk (Heisel, 2006). Age-related losses and transitions can induce narcissistic injury, threaten an older adult's perceived autonomy and control, and thus contribute to risk for suicide.

Methods: We investigated cross-sectional and longitudinal associations between perceived autonomy and suicide ideation in 173 community-residing older adults recruited into a prospective 2-year study of the onset and/or exacerbation of suicide ideation. Participants completed a demographics questionnaire and measures of suicide ideation, depressive symptom severity, self-rated health, and perceived autonomy at baseline, 2-4 week, 6-12 month, and 1-2 year follow-up assessments.

Results: Perceived autonomy was negatively associated with suicide ideation at baseline, controlling for participant sex, age, health ratings, and depressive symptom severity $(\Delta \mathrm{F}(1,164)=5.25, p=.023)$. Longitudinal findings supported a hypothesized interaction of baseline perceived autonomy and self-rated health on the onset or exacerbation of suicide ideation over 1-2 years of follow-up.

Conclusions: These, and associated findings, lend credence to the hypothesis that health-related transitions and losses may be especially pernicious to older adults' mental health and well-being and contribute to the onset of suicide ideation, and support efforts to enhance psychological resiliency among older adults struggling in the face of life transitions and attendant anticipated loss of autonomy and personal control.

Investigating Psychological

Risk and Resiliency to the

2b. Desire to Hasten Death and

Suicide Ideation among

Community-Residing

Older Adults

Tuesday

10:30 a.m.

Carlyle

Marnin Heisel, PhD, $\mathrm{CPsych}^{1}$, Gordon Flett, $\mathrm{PhD}^{2}$, Gil Schreier, MD, FRCP(C) ${ }^{3}$, Sharon Moore, RN, PhD, $\mathrm{CPsych}^{4}$

${ }^{1}$ Department of Psychiatry, Western University; ${ }^{2}$ York University; ${ }^{3}$ London Health Sciences Centre and Western University; ${ }^{4}$ Athabasca University

\section{Learning Objectives:}

1. Participants will learn about factors associated with suicide ideation and the wish to hasten death among community-residing older adults.

2. Participants will learn about overlap between the constructs of suicide ideation and the desire to hasten death as it impacts the aging Canadian population.

3. Discussion will focus on the implications of the present study for identification of risk for premature mortality among older adults and opportunities for sensitive care.

Background: Older adults have high rates of morbidity and mortality, including by suicide. Although the notion that most suicidal older adults are terminally ill is incorrect (Kleespies et al., 2000), receiving a terminal prognosis can induce suicide ideation (SI). Such thoughts subside quickly for most; yet, a proportion of terminally ill older adults continues experiencing a desire to hasten death (DHD; Chochinov, 2006), increasing risk for suicide. Research is needed investigating 
patterns of risk and resiliency to suicide ideation and the desire to hasten death in later life.

Methods: We recruited 173 community-residing older adults into a 2-year longitudinal study of the onset and/or exacerbation of suicide ideation. Participants completed measures of DHD (a seniors' version of the Schedule of Attitudes toward Hastened Death or SAHD; Rosenfeld et al., 1999) and SI (our Geriatric Suicide Ideation Scale or GSIS; Heisel \& Flett, 2006), and psychological risk (depression, social hopelessness, loneliness, daily hassles, and anxiety) and resiliency factors (meaning in life, subjective well-being, perceived social support, and psychological well-being).

Results: DHD was associated positively with GSIS items assessing Loss of Personal and Social Worth, and marginally with suicide ideation totals. DHD and SI were each associated positively with depression, hopelessness, loneliness, hassles, and anxiety, and negatively with meaning in life (MIL), subjective well-being, social support, and psychological well-being. Dimensions of MIL predicted significant variance both in 2-year DHD and SI, controlling for the severity of intervening depression and daily hassles.

Conclusions: Study findings thus support overlap between DHD and SI constructs, positive association of DHD with psychological risk factors and negative association with resiliency factors. Replication of these findings is needed with terminally ill older adults. Study findings will be discussed in the contexts of mental health-care needs for older adults at the end of life and clinical decision-making when working with older adults expressing a wish to hasten death.

\begin{tabular}{|c|c|c|}
\hline 2c. & $\begin{array}{l}\text { Meaning-Centred Men's } \\
\text { Groups for Men Facing } \\
\text { Retirement: A Preventive } \\
\text { Intervention to Reduce Risk } \\
\text { for Suicide }\end{array}$ & $\begin{array}{l}\text { Tuesday } \\
\text { 10:30 a.m. }\end{array}$ \\
\hline
\end{tabular}

Marnin Heisel, PhD, C.Psych ${ }^{1}$, Gordon L. Flett, PhD ${ }^{1}$, Paul S. Links, MD, FRCP(C) ${ }^{1}$, Ross M.G. Norman, PhD, CPsych $^{1}$, Sisira Sarma, $\mathrm{PhD}^{1}$, Sharon L. Moore, PhD, RN, RPsych ${ }^{1}$, Norm O'Rourke, PhD, RPsych ${ }^{1}$, Rahel Eynan, PhD $^{1}$, Kim Wilson, MSW, PhD(C) ${ }^{2}$, Paul Fairlie, $\mathrm{PhD}^{3}$, Beverly Farrell, R/TRO ${ }^{4}$, Kristan Harris, OT Reg. (Ont.) MHSc CHE ${ }^{4}$, Michelle Kerr, ${ }^{5}$, Bonnie Schroeder, MSW, $\mathrm{RSW}^{6}$ ${ }^{1}$ Department of Psychiatry, Western University; ${ }^{2}$ University of Guelph; ${ }^{3}$ York University; ${ }^{4}$ Third Age Outreach-St. Joseph's Health Care; ${ }^{5}$ London Kiwanis and Hamilton Road Seniors and Community Centre City of London; ${ }^{6}$ Canadian Coalition for Seniors' Mental Health

\section{Learning Objectives:}

1. Participants will gain familiarity with an innovative new intervention designed to enhance psychological resiliency and well-being, and decrease risk for the onset of depression, hopelessness, and suicide ideation among older men facing retirement.

2. Participants will learn about mental health challenges faced by older men facing retirement.

3. Participants will learn about meaning-centered approaches to enhancing mental health and well-being among older adults.

Background: Older men have the highest rates of suicide in North America and worldwide, accounting for over $80 \%$ of the nearly 9,400 North Americans over 60 who die by suicide every year. Few intervention studies have investigated suicide risk reduction among older adults to date, and nearly none has demonstrated significant reduction of suicide risk among older men. The present study describes a novel meaningcentered group intervention designed to enhance Meaning in Life (MIL) and prevent the onset of depression and suicide risk among men facing retirement.

Methods: We aim to recruit 100 community-residing men, 60 years and older, who plan to retire within the coming year or who have retired within the past year, into a 12-session course of meaning-centered group sessions designed to focus on transitions associated with retirement in the context of discussions about the meaning of work, retirement, leisure, relationships, and generativity. Eligible participants will be at potential risk for the onset of depression, hopelessness, and suicide ideation by virtue of facing retirement, a challenging life transition, and by endorsing extremely low levels of Meaning in Life (MIL), a key psychological resiliency factor shown to decrease risk for the onset of suicide ideation.

Results: The design, methods, and preliminary findings of this recently initiated 3-year, multi-centre study will be presented. Discussion will include focus on approaches we are implementing to recruit at-risk older men, group experiences, and initial findings investigating potential change in presence and severity of symptoms of depression, hopelessness, and suicide ideation and in MIL, perceived social support, and other psychological well-being variables.

Local Planning for

Psychogeriatric Services:

3a. Use of the Mental Health Commission of Canada Guidelines

Dr. Carole Cohen, $\mathrm{MD}^{1}$, Faith Malach, $\mathrm{MHSc}, \mathrm{MSW}, \mathrm{RSW}^{2}$ ${ }^{1}$ Sunnybrook Health Sciences Centre; ${ }^{2}$ Baycrest

\section{Learning Objectives:}

1. To showcase the use of the Mental Health Commission of Canada Guidelines for Comprehensive Mental Health Services for Older Adults in Canada in a local review and planning initiative for psychogeriatric supports. 
2. To highlight how the framework was utilized to inform recommendations for an ideal state of psychogeriatric supports in the TC LHIN.

3. To provide practical insights into the recommended benchmarks and alignment with local service provision.

Background: In January to March 2014, a Working Group was formed under the leadership of Baycrest to provide the Toronto Central Local Health Integration Network (TC LHIN) with a review of the current state of psychogeriatric resources providing support in long-term care homes and the community within the TC LHIN. It was recognized by the TC LHIN that there will be an increase in the number of seniors living in the TC LHIN and that a growing portion of those seniors will experience challenges related to mental health, dementia, and responsive behaviours. This includes those individuals who live in long-term care homes and within the community. Within the TC LHIN, there exist a number of psychogeriatric resource supports with opportunities and challenges related to access, effectiveness, capacity, integration, and efficiency. The current state of resources was presented and a critical analysis of resources was conducted for the TC LHIN. Further, the current state of psychogeriatric resources was compared to the Mental Health Commission of Canada (MHCC) proposed benchmarks and framework for comprehensive mental health services for older adults in Canada. Details on the use of the MHCC proposed benchmarks and framework and associated challenges and opportunities for use of the framework in planning will be presented.

Methods: The presentation will provide an overview of the Mental Health Commission of Canada Guidelines for Comprehensive Mental Health Services for Older Adults in Canada and illustrate how the guidelines and framework were used during analysis and planning for local recommendations focused on psychogeriatric supports.

Results: The presentation will include a description of themes and key questions encountered while engaging in the comparison of a current and ideal state for psychogeriatric services.

Conclusions: The use of a framework and guidelines for planning is useful as an approach to analyzing the current state of supports, and to guide planning and recommendations leading to transformation and investment in psychogeriatric supports.

Culture Change Toward

3b. Person-Centred Care in Long Term Care Homes

Mary Schulz, MSW, RSW

Alzheimer Society of Canada

\section{Learning Objectives:}

1. Raise awareness about the PC P.E.A.R.L.S. ${ }^{\mathrm{TM}}$ and the culture change initiative of the Alzheimer Society of Canada.

2. Learn practical strategies on how to begin and sustain culture change in long-term care homes.

3. Become culture change champions.

Background: In 2008, the Alzheimer Society of Canada embarked on a "culture change" initiative focusing on the needs of people with dementia living in long-term care (LTC) homes. Most people with dementia want to live in their own homes for as long as possible. However, many will move to a LTC home. $57 \%$ of seniors living in a residential care home have a diagnosis of dementia, and $70 \%$ of all individuals diagnosed with dementia will die in a LTC home.

Methods: The culmination of phase 1 was the launch in 2011 of the "Guidelines for Care: Person-Centred Care of People with Dementia Living in Care Homes". During phase 2 , an exploratory qualitative research was conducted to learn what is key in a "successful" culture change to person-centred care.

Results: In March 2014, based on these findings, we launched PC-PEARLS ${ }^{\mathrm{TM}}$ - seven information sheets providing key elements on how to begin and sustain culture change in long-term care.

Conclusions: Our most important objective is to ensure that a person-centred philosophy of care is well understood and put into practice in LTC homes to improve the experience of people with dementia, their caregivers, and the professionals supporting them. Our ultimate goal is to make person-centred care the norm rather than the exception in Canada.

Self-Reported Mood and

Subsequent Healthcare use in

Older Emergency Department Tuesday

4a.

Patients: Results of a

2:15 p.m.

Scott

Multicenter Prospective

Cohort Study

Aliya Ramjaun, $\mathrm{MSc}^{1}$, Samir Sinha, $\mathrm{MD} \mathrm{MSc}^{1}$, John Hirdes, $\mathrm{PhD}^{2}$, Andrew Costa, $\mathrm{PhD}^{3}$

${ }^{1}$ Geriatrics Research Program, Mount Sinai Hospital; ${ }^{2}$ University of Waterloo; ${ }^{3} \mathrm{McMaster}$ University

\section{Learning Objectives:}

1. Attendees of this presentation will appreciate the link between mood symptoms in the emergency department and other health measures and future health-care use; mood symptoms may prompt more efforts to ensure continuous primary care beyond the emergency department.

2. Audience members will learn that individuals with mood symptoms are more likely to return to the emergency 
department at a greater rate, indicating an issue of increased perceived severity of disease (physical or otherwise).

Background: Depression in later life is associated with disability, mortality, and poorer health-care-related outcomes. We sought to determine whether mood in the ED can predict unplanned health service use (return ED visits, hospitalization, discharge to alternate level care, comprehensive geriatric assessment (CGA)).

Methods: A multi-center prospective cohort study was conducted. Mood symptoms including depression, anhedonia, and anxiety were assessed. Patients were followed up for 90 days to determine frequency and dates of subsequent visits, hospitalizations, and discharge to alternate level care facilities. Whether an individual required CGA was noted at the index visit. Associations between mood and geriatric syndromes were assessed using chi tests for categorical variables. One-way analysis of variance was used to assess the association between mood symptoms and continuous variables. A series of univariate logistic regressions were performed to quantify the impact of mood on experiencing each outcome.

Results: 2,101 patients were included. $42.60 \%$ were discharged home, and of these 346 (39.54\%) returned to the ED. $1,079(52.53 \%)$ were admitted to hospital at the index ED visit, and $224(10.91 \%)$ were discharged to either alternate level or long-term care. Nearly all domains assessed demonstrated a significant association with mood, with the exception of sex, cognition, comprehension, and traumatic injury. Mood also emerged as a significant predictor of hospitalization at the time of the index ED visit (OR 1.19, 95\% CI 1.07-1.31), even after adjustment for the impact of comorbidities noted at the time of the index ED visit.

Conclusions: A number of associations exist between geriatric syndromes and the presence of self-reported mood symptoms amongst older ED patients. Mood may also impact the perceived severity of a given physical illness or presenting complaint and, therefore, the decision to hospitalize a patient.

\section{Delirium Prevention, Screening}

and Management from

Emergency Department to

Tuesday

Discharge Follow-up
Scott

William A. Nisker, MD, FRCP(C) ${ }^{1,2}$, Lily Spanjevic, RN, BScN, MN, GNC(C), CRN(C), CMSN(C) ${ }^{1}$, Trish Corbett, RN, BN, MN, GNC(c) ${ }^{1}$

${ }^{1}$ Joseph Brant Hospital; ${ }^{2} \mathrm{JBN}$ Medical Corporation

\section{Learning Objectives:}

1. To review evidence for assessment, prevention, and management targeting avoidance of prolonged delirium.

2. To understand the impact of delirium superimposed on dementia and its long-term effects.

3. To identify interventions to mitigate delirium in an acute care setting.

Background: Joseph Brant Hospital has 224 beds, 75.5\% of the in-patients are over the age of 65 and $42.9 \%$ are $>80$ years. One in four patients over the age of 85 has dementia the number one risk factor for delirium. Delirium prevalence in medical in-patients over the age of 70 is $30-40 \%$, in ICU is $70-80 \%$, hip fracture and CABG $45-55 \%$, (Marcantonio, 2010). Delirium increases length of stay, functional and cognitive decline, restraint use, admission to LTC, and morbidity. With the growing geriatric population, it is imperative to prevent and decrease delirium rates. This presentation will focus on a community hospitals approach to delirium management.

Methods: A multi-faceted approach was used to improve the quality of care of our geriatric patient population. A Geriatric Consult Team was developed to support implementation best practice at the bedside. A Senior Friendly Hospital (SFH) group was established to identify gaps in practice and conduct a needs assessment. A Chemical Restraint Working group was developed to review the literature, identify four key populations who were high users of chemical restraints; they created best practice policies and patient order sets. The SFH group identified a priority to implement a hospital-wide mobility and create discharge bundles to reduce recidivism. Community engagement to recruit volunteers for Hospital Elder Life Program (HELP) and Eating Matters Program.

Results: A revised Delirium Order Set, CAM tool, CAM-ICU tool, BPSD guidelines, Management of Acute Psychosis in the Emergency Department, and revised CIWA was developed. HELP implemented in Medicine and ED. Eating Matters Program improved patient intake. Stronger collaboration with CCAC and Mental Health Outreach Program resulted in better communication and access to services. Increased number of staff trained in Gentle Persuasive Approaches.

Conclusions: Delirium prevention, assessment, and effective management can be implemented in a busy acute care environment if a focused delirium strategy is implemented.

Collaborative Care Rounds

5a. Mental Health and Residential Care Outcomes

Christine Dobbelsteyn, BPsych, MSc (Gerontology) ${ }^{1}$, Nirmal Kang, MD, FRCP ${ }^{1}$, Lina Furminger, RN ${ }^{1}$, Sue Hundal, $\mathrm{RN}^{2}$

${ }^{1}$ Fraser Health Authority; ${ }^{2}$ Normanna and Dania Residential Care

\section{Learning Objectives:}

1. Understand the model of collaborative care rounds between geriatric mental health and residential care. 
2. Examine the data collected showing positive outcomes for both staff and residents.

3. Obtain information and tools to start collaborative care rounds and replicate the study.

Background: As a busy community Geriatric Mental Health Outreach team with 12 residential care facilities in Burnaby, $\mathrm{BC}$, we explored other options of service delivery in residential care settings resulting in collaborative care rounds.

Methods: Pilot study completed at Normanna Care Home with pre- and post-survey's measuring knowledge and comfort level of caring for seniors with mental health disorders. Other clinical indicators also collected such as use of psychotropics, aggressive incidents, and WCB claims.

Results: Increased knowledge and comfort level of staff. Decreased formal referrals to geriatric mental health programs with decreased workload. Decreased use of psychotropics, and decreased incidents of aggression between staff and residents and resultant decreased WCB claims.

Conclusions: Collaborative care rounds once a month in residential care settings is an effective mechanism in meeting the care needs of seniors in residential care presenting with mental health concerns.

Eliminating Stigma: A Focus on

5b. Seniors' Mental Health Bonnie Schroeder, Samantha Kolapak

Bonnie Schroeder, MSW, RSw ${ }^{1}$, Jennifer Dotchin, MA, PMP ${ }^{2}$ ${ }^{1}$ Canadian Coalition for Seniors' Mental Health; ${ }^{2}$ Mental Health Commission of Canada

\section{Learning Objectives:}

1. Encourage self-awareness regarding discrimination and stigma with health-care professionals who work with older adults with mental health issues.

2. Improve attitudes and behaviours of health-care professionals who work with older adults with mental health issues.

3. Improve the lives of seniors with mental health issues and their caregivers.

Background: This paper explores stigma experienced by older adults living with a mental health problem or illness, as well as strategies to encourage self-awareness regarding discrimination and stigma, and improve attitudes and behaviours of health-care professionals who work with older adults with mental health issues.

Methods: This paper will also highlight the results of the pilot of an anti-stigma workshop based on the evidence (research, practice, and lived) and the inclusion of a social contact videos to introduce people to lived experience.
Results: Participant feedback demonstrated a willingness to engage in the process of reducing stigma and found the personal stories through video to be particularly useful to enhance connections with older adults living with mental illness. Four identifiable themes emerged from the qualitative research in analyzing the participants' feedback including language, awareness, context, and seeing the person. Quantitative results showed a small overall improvement in Opening Minds Stigma Scale for Health Care Providers (OMS-HC) stigma scores among workshop participants from pre- to post-intervention. An examination of score changes for the scale's three main dimensions of stigma (attitudes, disclosure/help seeking, and social distance) showed that the largest reduction occurred on the 'attitudes towards people with a mental illness' subscale at a $6.5 \%$ improvement in score from pre- to post-workshop, which is comparable to other successful programs evaluated.

Conclusions: With an aging population and significant focus on seniors' mental health, this presentation provides an overview of the successful workshop material developed related to seniors' mental health issues and stigma.

Managing BPSD in the
6a. Acute Care Hospital Setting: Wednesday Scott
Scoping the Problem and $2: 15$ p.m.
Testing Solutions

Lesley Wiesenfeld, MD, MHCM, FRCPC ${ }^{1}$, Jocelyn Bennett, RN, $\mathrm{MSc}^{1}$, Kiran Rabheru, MD, CCFP, FRCPC ${ }^{2}$, Dallas Seitz, MD, FRCPC ${ }^{3}$, David Harvey ${ }^{4}$

${ }^{1}$ Mount Sinai Hospital, University of Toronto; ${ }^{2}$ The Ottawa Hospital; ${ }^{3}$ Providence Care Mental Health Services; ${ }^{4}$ Alzheimer Society of Ontario

\section{Learning Objectives:}

1. To develop appreciation for the opportunities and challenges providing safe patient care to patients with dementia in the acute hospital sector.

2. To learn about a variety of quality improvement-informed interventions to address the challenge of supporting patients with dementia who are admitted for medical or surgical care in the acute care hospital.

3. To develop strategies for both assessing and developing key metrics and deliverables to guide efforts to improve psychogeriatric patient care in the hospital setting.

Background: Given the prevalence of dementia and the aging population, an increasing number of patients admitted to the acute hospital setting have a diagnosis of dementia. Patients with dementia, especially those patients with behavioural and psychological symptoms of dementia, may be particularly vulnerable in the hospital setting. Both cognitive and noncognitive features of dementia may make it more challenging to deliver medical or surgical interventions. Furthermore, patients with dementia may become distressed or agitated in 
unfamiliar surroundings with change to their routine. The additional risk of hospital-acquired delirium further complicates the hospitalization of patients with dementia.

Methods: Hospitals vary in their approaches to responding to the care and safety needs that emerge from the presence of co-morbid dementia.

Results: In this presentation, the audience will learn how a variety of acute hospitals are approaching and scoping the clinical challenge and responding to it through innovative approaches to training, screening, and protocolized interventions.

Conclusions: Developing expertise and effectiveness responding to the needs of hospitalized patients with dementia requires application of quality improvement techniques and institutional leadership and support to develop and evaluate potential interventions with a view to developing best practice approaches.

\begin{tabular}{ll}
\multicolumn{1}{l}{ Innovation in Behaviour } & \\
6b. Support: Engaging and & Wednesday Scott \\
Enabling Innovation from & $2: 15$ p.m. \\
Front-Line Staff &
\end{tabular}

Janet Chee, RN, BScN, MN, PMP, Dorothy Huang, RN, Ivy Cheung, RPN Yee Hong Centre for Geriatric Care

\section{Learning Objectives:}

1. Learn how to utilize transformational leadership principles to create a culture of innovation.

2. Learn key innovations that were developed and the outcomes related to resident improved quality of life.

3. Learn the key success factors to both develop and sustain a culture of innovation.

Background: Canada is facing a significant aging population with seniors constituting the fastest growing population group. As a result, there are increasing numbers of seniors with cognitive impairments due to mental health problems, addictions, dementia, or other neurological conditions who often exhibit responsive or challenging behaviours such as aggression, wandering, physical resistance, and agitation in long-term care homes.

Methods: Yee Hong Centre for Geriatric Care joined the Behavioural Support Ontario (BSO) Project in 2012. The purpose of the BSO Project is to enhance services for elderly people with complex behaviours through the development and implementation of new models of care that focus on quality of care and quality of life. The implementation of this program has involved the utilization of a transformational leadership approach that has enabled the development of innovations from front-line staff that have enhanced the quality of life of Yee Hong residents.
Results: Innovations developed by front-line staff include the creation of a store experience that has brought to life a vivid shopping experience for dementia residents, the utilization of color in uniforms to encourage recognition of behavioural support staff, the streamlining of behaviour documentation, and the utilization of cultural competence to ensure innovations are rooted in resident culture.

Conclusions: Participants who attend this session will learn about developing a culture of innovation, hear about the innovations that have been developed by front line staff, as well as the outcomes of those innovations, and finally, learn about the key success factors that are critical not only to develop a culture of innovation but also to sustain it.

An e-Learning Program

about Behavioural and

Psychological Symptoms of

Wednesday

2:15 p.m.

Scott

Dementia (BPSD)

Marie-Andrée Bruneau, MD MSc FRCPC ${ }^{1}$, Caroline Ménard, MA ${ }^{2}$ ${ }^{1}$ University of Montreal; ${ }^{2}$ Institut Universitaire de Gériatrie de Montréal

\section{Learning Objectives:}

1. Describe the educational needs about BPSD.

2. Discover a new pedagogic medium (e-learning modules) developed in Quebec in order to increase staff's knowledge about BPSD.

3. Develop leadership in order to implement educational training program about BPSD around Canada.

Background: Various organizations have highlighted the access difficulties to specialized services for the management of behavioural and psychological symptoms of dementia (BPSD). Training needs of staff on the topic of BPSD are well documented; the authors of Quebec Alzheimer's plan deplore the weakness of the training offered in that respect. This gap leads to suboptimal management of BPSD and too much use of psychotropics.

Methods: It has been shown that staff training was the cornerstone in the prevention and intervention for BPSD. Whereas $80 \%$ of the clientele of long-term care suffers from dementia, these training needs are increasing. The recent deployment by the Quebec's Ministry of Health of clinical projects involving the first-line partners in collaboration with experts from memory clinics and BPSD teams stresses the need to implement such a teaching program. Therefore, the BPSD IUGM team, in collaboration with partners from Laval, McGill, Montreal, and Sherbrooke Universities, decide to create an e-learning program in order to increase professional knowledge and skills in the prevention and management of BPSD.

Results: Formal teaching methods have not been shown to be cost-effective. It is difficult and costly for managers to 
withdraw staff from their usual tasks for 1 to 2 day-long formations and the integration of acquired knowledge in the daily routine is usually short-term.

Conclusions: We decided to use modern technology in order to build a program of 15-minute e-learning modules that could be used anytime - at home or at work, for example — during case discussion.

\section{Fountain of Health:} Bringing Mental Health

7a. Promotion into Clinical Practice

Meagan MacNeil, MD, FRCPC, Vanessa Thoo, MD

Dalhousie University

\section{Learning Objectives:}

1. Participants will be able to identify barriers to implementation of mental health promotional initiatives targeting seniors.

2. Participants will acquire strategies for incorporating primary prevention into mental health assessments.

3. Participants will discuss mechanisms of knowledge transfer and behaviour change in both clinicians and patients.

Background: The Fountain of Health (FoH) Initiative is a Nova Scotia mental health promotion effort highlighting five key mental health evidence-based preventative interventions found to reduce risks of mental and cognitive illness and disability. In this knowledge transfer/ translation project, clinicians seeing seniors and caregivers in secondary and tertiary care settings were challenged to utilize FoH resources including: 1) a website, 2) an educational video, and 3) a patient handbook.

Methods: An in-person and tele-education presentation on five key $\mathrm{FoH}$ messages was disseminated to mental health clinicians via the Nova Scotia Seniors' Mental Health Network. Measures included: 1) presentation quality (evaluation form), 2) knowledge transfer (16-point knowledge pre- and post-questionnaire), and 3) knowledge translation (clinician volunteers tracked utilization of $\mathrm{FoH}$ material over two months).

Results: 54 clinicians attended the $\mathrm{FoH}$ education session $(N=54)$. Return rates of the evaluation form were $77.8 \%$ $(n=42)$ and of pre- and post-survey were $68.5 \%(n=37)$. Overall presentation quality was "very good" to "excellent" (4. 4 on a 5-point scale.) On a 16-point scale $(n=37)$, there was a significant $(p<.0001)$ difference in survey knowledge scores before and after the presentation, with a mean pre-test score of $7.08(45 \%)$ compared to a post-test score that doubled to $14.25 / 16(90 \%$ correct). The average increase in score was 7.17 (95\% CI 5.92-8.42). Knowledge translation data collection is still in progress.
Conclusions: Preliminary results suggest the Seniors' Mental Health Network is an effective mechanism for knowledge transfer to mental health clinicians regarding content of seniors' mental health promotion information.

A Supported Self-Care

Intervention for

Middle-Aged and

Older Primary Care

7b. Patients with Depressive

Symptoms in Association

Wednesday

2:15 p.m.

Carlyle

with Chronic Physical

Conditions: A Randomized

Controlled Trial

Jane McCusker, MD, DrPH ${ }^{1,2}$, Martin G. Cole, MD $^{3,4}$, Mark Yaffe, BSc, MDCM, MCISc, CCFP, FCFP ${ }^{5,6}$, Erin Strumpf, PhD $^{1}$, Maida Sewitch, $\mathrm{PhD}^{7}$, Tamara Sussman ${ }^{7}$, Antonio Ciampi ${ }^{7}$, Eric Belzile ${ }^{7}$ ${ }^{1}$ Department of Epidemiology, Biostatistics and Occupational Health, McGill University; ${ }^{2}$ St. Mary's Research Centre; ${ }^{3}$ Department of Psychiatry, McGill University; ${ }^{4}$ Geriatric Psychiatry Division, St. Mary's Hospital Center; ${ }^{5}$ Department of Family Medicine, McGill University; ${ }^{6}$ St. Mary's Hospital Center; ${ }^{7}$ Department of Medicine, McGill University

\section{Learning Objectives:}

1. Describe components of chronic disease and depression self-care interventions.

2. Differentiate between supported and unsupported selfcare interventions for depression.

3. Understand effects of trained lay coaching on use of selfcare materials and depressive symptoms in middle-aged and older people with chronic conditions.

Background: Although self-care interventions (SCI) for depression using a variety of modalities can be effective, there is little evidence regarding: 1 ) their effectiveness in the middle-aged and older adults with chronic physical conditions; and 2) the benefits of providing support in the use of self-care tools. The objectives of this study were to assess the effects of providing support on: 1) adherence to a SCI; and 2) depression outcomes in this population, and 3) to examine whether these outcomes differed by age.

Methods: Eligible patients in family physician practices (40 years and older, English or French speaking, with a chronic physical condition or pain of 6 months or greater, at least mild depressive symptoms, and without severe cognitive impairment) were randomized to an intervention (supported) or control (unsupported) group. All participants were given a multi-modality depression self-care toolkit. The intervention group was offered weekly telephone support from a trained lay coach for 3 months and then once per month up to 6 months. 
Results: Of 223 patients randomized, 172 (77\%) completed the trial. At 3 months, compared to controls, the intervention reported significantly greater adherence to the self-care tools and lower PHQ-9 scores. At 6 months, the difference in depression severity was attenuated and no longer statistically significant. Older patients $(65+)$ had similar rates of adherence and outcomes.

Conclusions: Trained lay coaching improves intervention adherence. It improves the severity of depressive symptoms at 3 months, but this effect is not sustained at 6 months.

\section{Participe présent:}

développement et

validation d'un programme

visant à promouvoir

la participation

Wednesday Carlyle
2:15 p.m.

communautaire des ânés

vivant avec un problème de

santé mentale

Manon Parisien, OT, $\mathrm{MSc}^{1}$, Kareen Nour, $\mathrm{PhD}^{2}$, Alan Regenstreif, $\mathrm{MSW}^{1}$, Ginette Aubin, $\mathrm{PhD}^{3}$, Véronique Billette, $\mathrm{PhD}^{1}$

${ }^{1}$ CSSS Cavendish - Centre de recherche et d'expertise en gérontologie sociale; ${ }^{2}$ Direction de santé publique de l'Agence de santé et de services sociaux de la Montérégie; ${ }^{3}$ Université du Québec à Trois-Rivières

\section{Learning Objectives/Après cette présentation, les participants pourront:}

1. Décrire les différents volets du programme Participe présent qui vise à promouvoir la participation communautaire des aînés vivant avec un problème de santé mentale.

2. Relater les principales étapes de la démarche de co-construction d'innovations sociales utilisée pour développer ce programme de promotion de la santé.

Background: Les problèmes de santé mentale (PSM) sont associés à la stigmatisation des aînés, à des répercussions sur leur santé et à plusieurs facteurs de risque (pauvreté, itinérance, longévité réduite) (Fleury et al., 2012). Cette présentation décrit un programme visant à promouvoir la participation sociale des aînés vivant avec des PSM. Les étapes de développement du programme et le résultat de sa validation par un comité consultatif seront exposés.

Methods: Une démarche collaborative a sous-tendu le développement du programme. Les principales étapes sont: formation d'un comité consultatif, étude des besoins, recension d'écrits, élaboration du modèle logique, conception des outils du programme, validation des outils et mise à l'essai auprès de la population cible (en cours). La validation du programme a impliqué des intervenants, des chercheurs, des organismes communautaires et des personnes qui vivent avec des PSM.

Results: Le programme Participe-présent vise à renforcer les compétences personnelles en matière de participation sociale et à créer des conditions favorables dans la communauté. Il comporte quatre volets: 1) une entrevue individuelle inspirée de l'entretien motivationnel, 2) un atelier éducatif de groupe (7 rencontres); 3) un projet collectif de communication médiatique et 4) des visites d'organismes communautaires. Le programme peut être offert par des professionnels de la santé, des intervenants du milieu communautaire ou des pairs aidants, à des groupes de six à dix aînés vivant avec des PSM.

Conclusions: Après sa mise à l'essai, Participe-présent pourra être diffusé largement afin de mieux outiller les intervenants à promouvoir la participation sociale des aînés qui vivent avec un PSM.

\section{Predictors of Participation}

8a. in an In-patient Geriatric Rehabilitation Program

Wednesday 3:45 p.m.

Rossetti

Diana Nicolici, MD, FRCPC ${ }^{1}$, Saba Shahab ${ }^{2,3}$, Alva Tang ${ }^{2,3}$, Erind Dvorani $^{2,3}$, Joanna Schechter, BSc(PT), BA ${ }^{3}$, Peggy Arden, MSW, $\mathrm{RSW}^{3}$, Ruki Kondaj ${ }^{2,3}$, Nahid Kamsorkhi ${ }^{2,3}$, Paul Katz, MD, CMD, FRCPSC ${ }^{3}$, Linda Mah, MD, MHS, FRCPC ${ }^{2,3,4}$

${ }^{1}$ University of Toronto; ${ }^{2}$ Rotman Research Institute, Baycrest; ${ }^{3}$ Baycrest Centre for Geriatric Care; ${ }^{4}$ University of Toronto

\section{Learning Objectives:}

1. Be aware of the current evidence regarding clinical predictors for rehabilitation participation.

2. Learn about the impact of rehabilitation participation on functional outcome of geriatric in-patient rehabilitation.

3. Learn about the results of the Baycrest rehabilitation study on predictors of rehabilitation participation.

Background: An important determinant of functional outcome following rehabilitation is the degree to which patients participate during therapy. In the current study, we sought to identify clinical predictors of elderly patients' ability to participate in an in-patient rehabilitation program.

Methods: The sample included 69 patients [mean age 82 (SD9.9); $\mathrm{F}: \mathrm{M}=1.55]$ admitted to a University-affiliated general in-patient rehabilitation program. Consenting patients were assessed using measures of mood, pain, cognition (Montreal Cognitive Assessment [MoCA]), and overall function (Functional Independence Measure [FIM]) as potential predictors of participation. Participation in therapy sessions was evaluated using the Pittsburgh Participation Scale (PPS) and Hopkins Rehabilitation Engagement Rating Scale (HRERS). Multiple regression models were used to determine predictors of PPS and HRERS outcomes.

Results: A multiple regression model to determine predictors of participation as assessed using the PPS identified only FIM and MOCA as significant factors (standardized $\beta=.29, p=$ .02 and $\beta=.26, p=.04$, respectively) and accounted for $36 \%$ of the variance $(\mathrm{F} 5,51=5.94 ; \mathrm{R} 2=.36, \mathrm{p}<.001)$. A multiple 
regression model to determine predictors of participation as assessed using the HRERS identified only FIM as a significant predictor (standardized $\beta=.36, p=.004$ ) and accounted for $33 \%$ of the variability $\left(\mathrm{F} 5,51=5.10 ; \mathrm{R}^{2}=.33, p<.001\right)$.

Conclusions: Cognitive status appears to be an important determinant of elderly patients' ability to participate in inpatient rehabilitation. However, our models accounted for only one-third of the variability in extent of participation. Therefore, other potential predictors need to be identified. Further, it will be important to identify which specific cognitive deficits contribute and the mechanisms whereby these deficits mediate older adults' ability to participate in rehabilitation.

Modified Mindfulness-

Based Cognitive Group

$8 b$.

Therapy with Cognitively Impaired Elderly

Anyssa Shakeri, MD

University of British Columbia

\section{Learning Objectives:}

1. Understand a model of modified MBCT for cognitively impaired elderly.

2. Explore the benefits of modified MBCT for older adults.

3. Experience modified MBCT in session.

Background: The objective of our project is to trial modified mindfulness-based cognitive therapy (MBCT) techniques in a group setting for older adults with anxiety and depression in order determine their effects on quality of life. We wish to determine whether MBCT is suitable for older adults and the modifications required, particularly for cognitively impaired older adults.

Methods: This has been done by enrolling patients in an 8 -week mindfulness and relaxation group for older adults, which meets once a week for one hour. Group is facilitated using MBCT techniques with modifications. Patients are referred to the group via referral form to be completed by their community psychiatrist, family physician, or mental health team case manager. Inclusion to the group and project requires patients to be age 65 or greater, with a diagnosis of a depressive or anxiety disorder and attendance of $75 \%$ or greater in the sessions. Quality of Life Scales have been done pre- and post-group.

Results: We have noted an overall improvement in quality of life in study participants using the modified MBCT program.

Conclusions: We believe there are benefits to modified MBCT for cognitively impaired elderly and are developing a program to implement in other health authorities.
Grey Matter Volume

Predicts Accurate

Response Rate in Patients

8c. with Mild Cognitive

Impairment and Older

Controls during a

Working Memory Task

Jun Ku Chung, $\mathrm{HBSc}^{1}$, Amer Burhan, $\mathrm{MBChB}$, $\mathrm{FRCPC}^{2}$, Ariel Graff-Guerrero, MD, PhD ${ }^{3}$

${ }^{1}$ Institute of Medical Science, University of Toronto; ${ }^{2}$ Division of Geriatric Psychiatry, University of Western Ontario; ${ }^{3}$ Department of Psychiatry, University of Toronto

\section{Learning Objectives:}

1. Mild Cognitive Impairment definition and types.

2. Working memory definition and assessment.

3. Brain regions involved in working memory performance.

Background: Neuro-imaging studies report that frontoparietal-temporal brain regions modulate performance during working memory tasks (WMT). However, no information is available of the brain structures involved in accurate response during WMT. Therefore, the aim of our study was to investigate if gray matter volume (GM) in frontal, parietal, and temporal regions were associated with accurate response rate (ARR), during a WMT, in patients with mild cognitive impairment (MCI) and controls.

Methods: The sample was composed of only women, 10 with MCI ( $72.7 \pm 9.3$ years old) and 12 controls ( $65.8 \pm 6.8$ years old). The participants underwent a WMT during an fMRI session. ARR was estimated from the WMT for each participant. Voxel-based morphometry with DARTEL method was performed using participant's high resolution T1-weighted images. Voxel-wise correlation analysis was carried out between ARR and GM, considering frontal, parietal, and temporal regions as a priori. The complete sample was included in the analysis in order to maximize the variability in ARR and increase the likelihood of finding stable correlations.

Results: ARR showed positive correlation with bilateral middle frontal $(t=4.81, p$ (uncorrected) $<.001)$ and negative correlation with lateral temporal $(t=4.74, p$ (uncorrected) $<.001)$, and parahippocampus $(t=4.81, p$ (uncorrected) $<.001)$. No correlation was found between the total intracranial volume and ARR. Age was similar for both groups $(t=-1.97, p=.067)$. As expected, controls shower better ARR in comparison to patient with MCI $(t=6.956, p<.001)$.

Conclusions: Our pilot study suggests that fronto-temporalparahippocampal regions may modulate ARR during a WMT. Interestingly, increased frontal GM and decreased lateral temporal, and parahippocampal GM seem to be associated with better ARR. 


\section{WORKSHOP ABSTRACTS}

Future of Geriatric

Psychiatry Recruitment in

Canada - Discussion about

the Results of a Pan-Canadian 3:45 p.m.

Wren

Online Survey of Psychiatry

Residents

Soham Rej, MD ${ }^{1}$, Vincent Laliberte, MD, MSc ${ }^{1}$, Melissa Andrew, MD, MEd, FRCPC ${ }^{2}$, Catherine Shea, MD, FRCPC ${ }^{3}$, Marla Davidson, MD, FRCPC ${ }^{4}$, Tricia Woo

${ }^{1}$ McGill University; ${ }^{2}$ Queen's University; ${ }^{3}$ University of Ottawa; ${ }^{4}$ University of Saskatchewan

\section{Learning Objectives:}

1. To learn about an online survey of Canadian psychiatry residents that examined predictors of interest in geriatric psychiatry.

2. To hear from a panel of leading clinician-educators in geriatric psychiatry and their opinion about the survey's results.

3. To engage in a discussion about future strategies to improve recruitment and training of psychiatry residents in geriatric psychiatry.

Background: In spite of a pending "silver tsunami", there remains a shortage of Canadian geriatric psychiatrists. The demographic, educational, and vocational predictors of residents' interest in geriatric psychiatry have not yet been clearly elucidated.

Methods: We conducted a Canada-wide, Web-based, crosssectional survey, which aimed to ascertain what percentage of Canadian psychiatry residents are interested in: a) becoming geriatric psychiatrists, b) obtaining FRCPC geriatric psychiatry certification, and c) working with older adults as part of their practice. Bivariate and multivariate analyses were performed to better understand what demographic, educational, and vocational variables are associated with interest in becoming a geriatric psychiatrist.

Results: 207/853 (24.3\%) of all psychiatry residents responded to the survey. $29 \%$ of respondents $(n=60)$ had either an interest or a strong interest in becoming a geriatric psychiatrist. Bivariate analyses revealed that, although demographic factors were not associated with interest in geriatric psychiatry, certain educational variables (e.g., early and positive training experiences in geriatric psychiatry) and vocational factors (e.g., enjoyment of working with complex multi-factorial clinical issues, including physical health issues) were important predictors of interest. Multivariate analyses will be completed by mid-April 2014 and will be ready for CAGP 2014 .

Conclusions: This workshop will involve a brief introduction of the topic by co-chairs Soham Rej and Dr. Marla Davidson, followed by a 15-minute presentation of the survey's data by Vincent Laliberte. After that, Dr. Tricia Woo (geriatric medicine), Dr. Davidson, Dr. Melissa Andrew, and Dr. Catherine Shea (geriatric psychiatry) will comment on the results and potential implications for geriatric education in Canada in the context of the field's recent FRCPC subspecialization status. The second half of the workshop will be dedicated to interaction with the panel and to collaboratively brainstorm new strategies to address the shortage of geriatric psychiatrists in Canada.

Promoting the Mental
W2. Health of At-Risk Seniors
through Inter-Disciplinary
Interventions Roundtable

Simone Powell, MSW, MPA ${ }^{1}$, Penny MacCourt, MSw, $\mathrm{PhD}^{2}$, Patti Gorr, $\mathrm{BA}^{1}$

${ }^{1}$ Public Health Agency of Canada; ${ }^{2}$ Thompson Rivers University

\section{Learning Objectives:}

1. Participants will enhance their skills in delivering mental health promotion to older adults by exploring intervention strategies and sharing information about relevant tools and resources.

2. Health professionals will explore their unique role in mental health promotion and will better understand the perspectives of other disciplines.

Background: Most adults enjoy good mental health throughout their lives. Seniors with or without mental health illnesses may experience challenges to their mental health associated with physical changes, chronic diseases, changes in social support networks, and low income. Those who are socially isolated, frail and/or living with dementia can be particularly at risk. With the aging of the population, there will be more seniors at risk for mental health problems. Given the range of risk factors for poor mental health, multi-sectoral/disciplinary approaches to mental health promotion are crucial.

Methods: The Mental Health Commission of Canada identified seniors as a priority for promotion and prevention activities emphasizing the need to: build capacity to promote mental health in later life; prevent mental illness and suicide; and intervene early when problems first emerge. Recommended actions included addressing stigma and helping older adults participate in meaningful activities, sustain relationships, and maintain good physical health.

Results: This roundtable discussion will identify of effective interventions and approaches that have been implemented at the community level and an enhanced understanding of the conditions which promote mental health. Mental health promotion interventions can target individuals, organizations, communities, and/or society and include interventions 
which: create supportive environments; develop personal skills; provide support for everyday activities; provide antistigma training; design effective programs and policies by implementing the Seniors' Mental Health Policy Lens; and/ or target structural barriers.

Conclusions: Participants will learn about initiatives addressing mental health promotion and share examples of policies, programs, and/or interventions that support seniors to maintain mental health despite potential challenges.

$\begin{array}{ll}\text { The BC BPSD Algorithm } & \\ \text { W3. - an Introduction to its } & \text { Tuesday } \\ \text { Development and Practical } & 3: 45 \text { p.m. } \\ \text { Application }\end{array}$

Carol Ward, MD FRCP(C) $)^{1,2}$, Elisabeth Drance, $\left.\operatorname{MD~FRCP(C)}\right)^{3,2}$

${ }^{1}$ Tertiary Mental Health Interior Health Authority; ${ }^{2} \mathrm{UBC}$;

${ }^{3}$ Parkview Intensive Behaviours Support Unit, Vancouver Coastal Health Authority

\section{Learning Objectives:}

1. The participants will learn to use the BC BPSD interactive Web-based algorithm in providing assessment and treatment for patients.

2. The participants will learn about guideline-recommended pharmacotherapy for BPSD.

3. The participants will develop an appreciation for the collaborative, interdisciplinary process involved in developing this provincial algorithm.

Background: In 2012, the BC provincial government released a Best Practice Guideline for Accommodating and Managing Behavioural and Psychological Symptoms of dementia for residential care. While the original goal for the guideline was to decrease the numbers of prescriptions for antipsychotics, the need to review and outline evidence-based practices in non-pharmacological and pharmacological care for elders with responsive behaviours was recognized as essential.

This guideline included an Algorithm for Accommodating and Managing Behaviours in Dementia developed by Dr. Carol Ward and Elisabeth Antifeau from Interior Health Authority (IHA) that incorporated the P.I.E.C.E.S. framework and medication usage, as well as IHA specific tools. After publishing the Best Practice Guide, the Algorithm was further developed to become a Web-based, practical, provincial tool; this was achieved by bringing representatives together from each health authority to review it and contribute tools, thereby developing a consensus document for the province. The BC Patient Safety and Quality Council provided the technical support.

The target users of the algorithm are staff and family practitioners working in residential care. This tool has a specific focus on non-pharmacological interventions and the appropriate use of antipsychotics in residential care. P.I.E.C.E.S. is integrated into the algorithm, which also includes an SBAR for BPSD, examples of person-centred care plan, a focus on least restraint, and guidance for prescribing psychotropic medications when needed. The algorithm can be bookmarked to a smart device for portability (www.bcbpsd.ca).

A clinical case will be used to demonstrate the Webbased algorithm to the participants.

$\begin{array}{ll}\text { W4. Violence Risk Assessment } & \text { Tuesday } \\ \text { for Geriatric Psychiatrists } & 3: 45 \text { p.m. Rossetti }\end{array}$

Zohar Waisman, MD, FRCPC ${ }^{1,2}$, Goran Eryavec, MD, FRCPC ${ }^{1}$

${ }^{1}$ North York General Hospital; ${ }^{2}$ Ontario Shores Centre for Mental Health Sciences

\section{Learning Objectives:}

1. To review of demographics and prevalence of violent behaviours by geriatric patients.

2. To learn basic principles, actuarial versus clinical assessment-how to use these principles in making clinical decisions re. transfer to nursing home, long-term care, home, or acute care setting.

3. To apply risk management techniques to risk assessment decisions.

Background: Violence in nursing homes, acute care settings, and long-term settings in geriatric patients are a reality that cannot be ignored; however, geriatric psychiatrists may not be familiar with the advances in risk assessment and management that are often used by forensic psychiatrists. The purpose of this workshop is to establish a collaborative framework through which better decisions can be made regarding risk assessment and management of the geriatric patient.

Methods: The workshop will teach the use of common actuarial measures of violence risk, and review the clinical assessment of violence as pertaining to this patient population through case examples and interactive format discussions.

Results: The case examples will be reviewed and scoring results will be distributed.

Conclusions: The participants will learn practical tools that could be applied immediately upon course completion to their daily work.

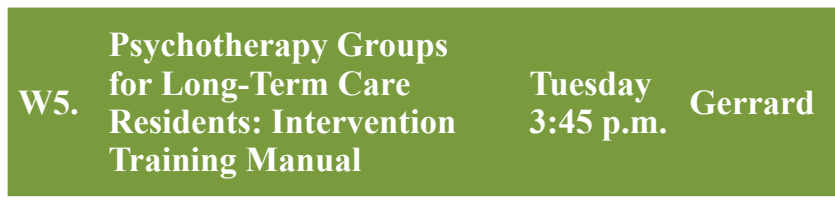

Kenneth Schwartz, MD, FRCPC

Baycrest 


\section{Learning Objectives:}

1. Describe the benefits of psychotherapy groups designed exclusively for the most cognitively intact long-term care (LTC) residents.

2. Identify the challenges associated with conducting these groups in long-term care settings.

3. Apply knowledge of the model with the intent of starting similar groups in LTC facilities.

Background: When individuals find themselves requiring LTC admission, they arrive under most vulnerable circumstances. ${ }^{(1)}$ However, the psychotherapeutic needs of nursing home residents remain underserved and poorly studied. ${ }^{(2)}$ It is especially difficult for more cognitively intact residents to access psychological help. ${ }^{(3)}$

Methods: A naturalistic study demonstrating the use of a group psychotherapy intervention that addresses the mental health and psychosocial needs of the physically frail and relatively cognitively intact (though often still with mild cognitive impairment or symptoms of early mild dementia) an individual faces upon entry into a nursing home is described. Throughout the study, the 12 weekly group psychotherapy sessions for six newly admitted individuals were audio-recorded, transcribed, and analyzed by the Clinician-Researcher and Research Assistant.

Results: This present study provides the first empirical insight into the suitability of the trans-theoretical method stage-of-change construct ${ }^{(4)}$ to adjustment behaviours in a LTC facility. ${ }^{(5)}$ A model of six stages of adjustment to both a nursing home and group is uniquely developed to track an individual's movement on stages of change. Major group themes and process will also be illustrated. A focus group to demonstrate the benefits of the group intervention serves as another form of a qualitative program evaluation.

Conclusions: Adjustment to a nursing home is a unique and challenging experience. ${ }^{(6)}$ The use of a brief supportive and integrated group therapy intervention can ease the stress of transition and help individuals to retain their autonomy and dignity.

1. Kao HS, Travis SS, Acton GJ. Relocation to a long-term care facility: working with patients and families before, dying, and after. J Psychosoc Nurs Mental Health Serv. 2004;42(3):10-16.

2. Bharucha AJ, Dew MA, Miller MD, et al. Psychotherapy in longterm care: a review. J Am Med Dir Assoc. 2006;7(9):568-80.

3. Schwartz K. Remembering the forgotten: psychotherapy group for the nursing home resident. Int J Group Psychother. 2007;57(4):497-516.

4. Prochaska JO, DiClemente CC. Stages and changes of selfchange of smoking toward integrative model of change. J Clin Consult Psychol. 1983;51(3):390-95.

5. Schwartz K. Psychotherapy training groups for long-term care residents: intervention training manual. Toronto: Baycrest; 2011.
6. Porter EJ, Kruzich JM. Rationale for individualizing interventions to promote adjustment to the nursing home. J Clin Geropsychol. 1999;5(2):79-90.

\begin{tabular}{|ll|}
\hline Innovation in Dementia & Wednesday \\
W6. Care: Therapeutic & $10: 15$ a.m. \\
Simulation for Caregivers & \\
\hline
\end{tabular}

Joel Sadavoy, MD, FRCP(C), FCPA, LJ Nelles, MFA, PhD, Virginia Wesson, MD, MSc, FRCP(C)

Mount Sinai Hospital

\section{Learning Objectives:}

1. Participants will be introduced to a unique therapeutic method that has a broad range of applications.

2. Participants will learn techniques for using therapeutic simulation with an elderly population.

3. Participants will reflect on possible application and integration at their own institutions or programs.

Background: In the Reitman Centre CARERS and Working CARERS Programs at Mt. Sinai Hospital, simulation is used in a focused group therapeutic intervention with family caregivers of people with dementia. While the process uses elements from validated simulation methodology, in therapeutic simulation mental health clinicians create a container that is different from an educational setting. Content for simulation is drawn directly from the caregiver's current experiences and enacted with a simulated patient. Relational habits and emotional factors are exposed and analyzed with the help of clinician facilitators and then a process is begun for changing behaviours, communication patterns, and attitudes.

Methods: Participants will learn about therapeutic simulation through video demonstration, participatory exercises, and facilitated discussion. Presenters will provide theoretical background and a demonstration of their methodology as a foundation. Participants will practice the methodology in facilitated small groups with standardized patients; discussion will include reflection and strategies for implementation. Introduction and demonstration (15 min); facilitated experiential practice (20 min); facilitated conversation including reflection and translation to practice (20 min); concluding discussion (5 min).

Results: Participants will be introduced to the concept, structure, practice, and clinical impact of therapeutic simulation as it is used in the Reitman Centre programs.

Conclusions: Participants will gain knowledge through practice of an effective therapeutic tool and will discuss methods to effectively integrate the demonstrated techniques within their own programs or institutions. 
W7. Psychosis in the Elderly: What's New?

Wednesday 10:15 a.m.

Carlyle

Corinne Fischer, MD, FRCPC ${ }^{1}$, Zahinoor Ismail, MD FRCPC ${ }^{2}$, Tarek Rajji, MD FRCPC ${ }^{3}$

${ }^{1}$ St. Michael's Hospital; ${ }^{2}$ Hotchkiss Brain Institute, University of Calgary; ${ }^{3}$ Centre for Addiction sand Mental Health

\section{Learning Objectives:}

1. To review the clinical presentation of psychotic disorders in the elderly, including psychosis in dementia and late onset psychosis.

2. To review the neurobiology of psychotic disorders in the elderly, including psychosis in dementia and late onset psychosis.

3. To review the management of psychotic disorders in the elderly, including investigations and treatment (nonpharmacological and pharmacological).

Background: Psychotic symptoms occur in approximately one-third of patients with dementia. In addition, it is estimated that $10 \%$ of patients with schizophrenia and related psychotic disorders develop their symptoms in late life, after the age of forty (late onset schizophrenia (LOS)). Psychotic symptoms are associated with adverse outcomes in dementia, while patients with LOS may have a more benign course compared to patients who develop symptoms early in life. Data on treatment of these symptoms in the elderly are limited and much has been extrapolated from studies conducted on nursing home populations or younger subjects.

Methods: This symposium will review the epidemiology and clinical presentation of psychotic disorders in the elderly, including dementia with psychosis and late onset psychotic disorders. In addition, we will review the neurobiology of these symptoms and discuss treatment approaches.

Results: This symposium will enhance the participant's understanding of psychotic disorders in the elderly in terms of their prevalence and clinical presentation. In addition, the participant will be exposed to the latest evidence in terms of treatment of these disorders.

Conclusions: Psychotic disorders in the elderly are encountered not uncommonly by geriatric psychiatrists. While their clinical presentation is complex, we are advancing in our understanding of the neurobiology of these symptoms, leading to improved treatment approaches and better outcomes.

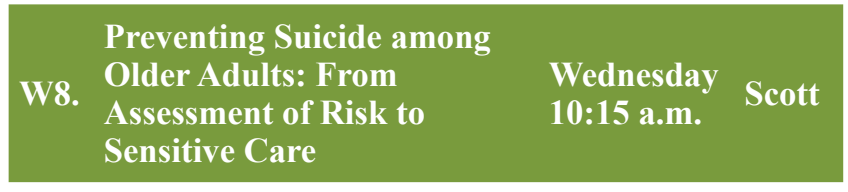

Marnin Heisel, PhD, $\mathrm{CPsych}^{1}$, Bonnie Schroeder, MSW, RSW ${ }^{2}$

${ }^{1}$ Department of Psychiatry, Western University; ${ }^{2}$ Canadian Coalition for Seniors' Mental Health

\section{Learning Objectives:}

1. Participants will gain familiarity with the Canadian Coalition for Seniors' Mental Health (CCSMH) late-life suicide prevention knowledge translation toolkit.

2. Participants will learn about the epidemiology of late-life suicide and associated risk, and resiliency factors and approaches to risk identification and intervention.

3. Interactive discussion will focus on methods for incorporating evidence-based tools to sensitive outreach, assessment, and intervention with older adults potentially at risk for suicide.

Background: Older adults have among the highest rates of suicide worldwide and typically utilize violent means of self-harm, necessitating development and dissemination of effective approaches to outreach, risk identification, and intervention. Yet, many at-risk older adults never receive recommended care, due in part to lack of knowledge among providers of evidence-informed approaches to risk detection and intervention. This issue is rapidly gaining prominence among health-care providers, researchers, policy analysts, and advocates, coincident with the tremendous growth in the North American older adult population and high rates of suicide in later life.

Responding to a need for older adult specific recommendations, and tools for enhancing health-care and social support for at-risk older adults, the Canadian Coalition for Seniors' Mental Health (CCSMH) has developed and broadly disseminated a late-life suicide prevention knowledge translation toolkit for front-line providers. The toolkit currently includes a set of national guidelines for suicide risk assessment and intervention, a clinician quick-reference card, an interactive training DVD, a guide for family members of at-risk older adults, and a guide for educators to facilitate training sessions.

This interactive workshop will focus on enhancing attendees' understanding of issues relevant to the advent and/ or reduction of suicide risk in later life, and will include discussion of the epidemiology of late-life suicide, associated risk and resiliency processes, and approaches to risk identification and assessment and to sensitive clinical and public health intervention.

We will additionally present findings of a CIHR-funded knowledge translation study in which we demonstrated enhanced provider knowledge and attitudes toward working with at-risk older adults. 


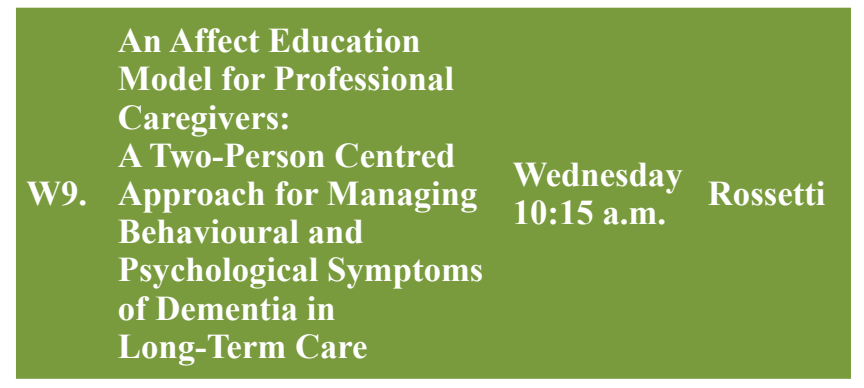

Kenneth Schwartz, MD, FRCPC Baycrest

\section{Learning Objectives:}

1. To identify how professional and family caregivers can respond in ways that elicit more problematic behaviours in individuals with BPSD.

2. To learn an innovative affect education model for caregivers.

3. To learn about a pilot study intended to lessen caregiver burden and which incorporates the use of the model.

Background: Treatment interventions for BPSD use nonpharmacologic and/or pharmacologic approaches. However, BPSD contributes to significant LTC staff burnout and turnover. $^{(1)}$ Woods $^{(2)}$ points out problems that may arise in caregiver's responses; stressed carers may behave in ways that elicit more difficult behaviours.

Methods: Funding has been obtained to study the effectiveness of an innovative Affect Education Model for Caregivers that addresses the issue of 'caregiver burden' of staff working on a LTC behavioural support unit. ${ }^{(3)}$ Nursing and ancillary staff will be taught the Affect Education Model ${ }^{(4)}$ in five sessions. Staff will learn, reflect, and discuss in a group format, and be encouraged to employ the approach in their work. A similar interactive approach will be employed in the workshop.

Results: Detailed case examples incorporating the use of Zeisel's Seven Questions Model ${ }^{(4)}$ that will be employed in educating nursing and ancillary staff working on a LTC behavioral support unit for individuals with BPSD will be illustrated. Preliminary quantitative and qualitative data will be presented.

Conclusions: Caregivers benefit from using a different approach when with a person with BPSD, one in which they can become reflective of their own feelings, leaving them to formulate a better approach. ${ }^{(5)}$ Zeisel's Seven Questions Model, developed to improve one's interpersonal relationships and adapted in this pilot study for the first time for use with individuals with BPSD, encourages caregivers to reflect on both their own feelings and feelings of others.
1. Pitfield C, Shahriyarmolki K, Livingston G. A systematic review of stress in staff caring for people with dementia living in 24 hour settings. Int Psychogeriatrics. 2011;23(1):4-9.

2. Woods RT. Discovering the person with Alzheimer's disease: cognitive, emotional and behavioral aspects. Aging Mental Health. 2001;5 (Suppl.1):S7-S16.

3. Zarit SH, Reevers KE, Bach-Peterson GJ. Relatives of the impaired elderly: correlates of feelings of burden. Gerontologist. 1980;20(6):649-55.

4. Zeisel E. Affect education and the development of the interpersonal ego in modern group psychoanalysis. Int J Group Psychother. 2009;59(3):421-32.

5. Talerico KA, Evans LK. Making sense of aggressive/protective behaviors in persons with dementia. Alzheimer's Care $Q$. 2000;1(4):77-86.

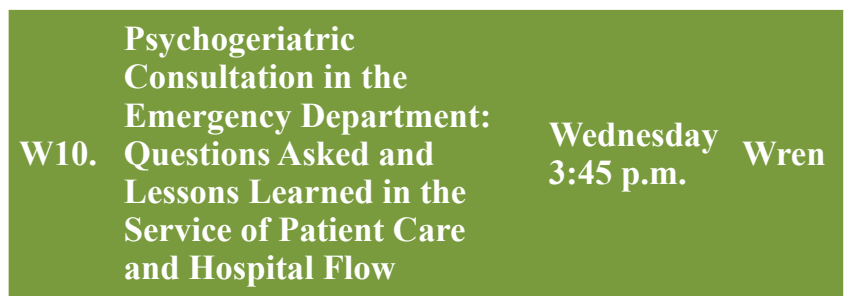

Lesley Wiesenfeld, MD, MHCM, FRCPC, Nana Asomaning, RN, NP, Mavis Afriyie-Boateng, RN, CNS

Mount Sinai Hospital, University of Toronto

\section{Learning Objectives:}

1. To understand the psychogeriatric needs and demands at the emergency department interface.

2. To develop an approach to efficiently and effectively providing dispositionally meaningful psychogeriatric consultation within the constraints of an emergency department visit.

3. To understand the acute care system flow implications of ER processes with respect to psychogeriatric patients.

Background: As the 'demographic tsunami' progresses, increasing numbers of patients and families are presenting to the acute hospital sector emergency department exclusively and explicitly for assistance related to psychogeriatric syndromes, most commonly dementia and/or delirium.

Methods: In response to the complexity of assessment and disposition challenges related to this patient population, both local and system-wide approaches are being considered to optimize patient care, as well as flow, through acute, community, and long-term care sectors. We will present one hospital's approach to responding to this clinical service challenge and describe an inter-professional approach to leverage nursing, and psychogeriatric and allied health engagement in the ED.

Results: Targeted, local investments in psychogeriatric resources in the ED and the development of collaborative, 
inter-professional protocols to harvest resources and guide ED assessment, have led to optimized dispositions, appropriate admission diversion, and early interventions for patients requiring acute hospital admission.

Conclusions: Front-line and front-loaded psychogeriatric engagement in the acute hospital emergency department can improve ED and hospital flow and contribute to responsive patient care.

A Systematic Approach
to the Treatment
W11. of Agitation and
$\begin{aligned} & \text { Aggression Associated } \\ & \text { with Dementia }\end{aligned}$

Benoit H. Mulsant, MD, MS ${ }^{1,2}$, Tarek K. Rajji, MD ${ }^{1,2}$, Simon Davies, $\mathrm{MD}, \mathrm{PhD}^{3}$, Donna Kim, $\mathrm{MD}^{3}$, Bruce Pollock, $\mathrm{MD}, \mathrm{PhD}^{3}$ ${ }^{1}$ Campbell Family Mental Health Research Institute, Centre for Addiction and Mental Health (CAMH); ${ }^{2}$ University of Toronto; ${ }^{3} \mathrm{CAMH}$

Learning Objectives: At the conclusion of this session, the participants should be able to:

1. Assess the evidence supporting the efficacy of pharmacotherapy for the treatment of agitation and aggression associated with dementia.

2. Assess the evidence supporting the efficacy of nonpharmacologic interventions for the treatment of agitation and aggression associated with dementia.

3. Discuss how these interventions can be integrated in a care pathway supporting a stepped-care strategy.

Background: Both pharmacologic and non-pharmacologic interventions have been found to be helpful in the treatment of older patients with dementia and agitation or aggression. However, when used under usual care conditions, these interventions yield outcomes that are typically suboptimal. During the past decade, systematic ("stepped care") approached embedding pharmacotherapy algorithms have been shown to improve the outcomes of late-life mental disorders. At the conclusion of this session, the participants should be able to assess the evidence supporting the efficacy of pharmacotherapy for the treatment of agitation and aggression associated with dementia.

Methods: In this workshop, the presenters will engage the audience to discuss: factors that impede good outcomes under usual care conditions; how a systematic approach to treatment can overcome these impediments; whether treatment algorithms ignore clinical wisdom and deprive patients from a personalized approach. They will also share their experience developing and implementing an algorithm-based integrated care pathway to optimize the treatment of agitation or aggression in older in-patients with dementia.
Results: Two principles guided the development of the pathway: it had to be based on the evidence; and it had to be practical, flexible, and accommodate local expertise. One group of psychiatrists developed the pharmacological algorithm; an overlapping inter-professional group developed the non-pharmacological arm and selected the assessments tools. The pathway consists of an assessment phase, a medications withdrawal phase, and then a treatment phase. The pharmacological algorithm consists of six steps. The nonpharmacological interventions comprise of both purposeful social activities and sensory modulations.

Conclusions: We have developed a pathway embedding an algorithm-based approach to treat agitation and aggression in in-patient settings. We will also present data about its implementation and associated outcomes.

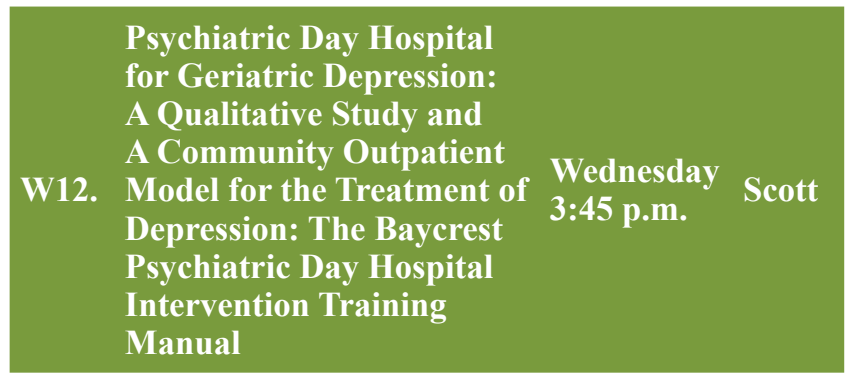

\section{Part 1: Psychiatric Day Hospital for Geriatric De- pression: a Qualitative Study}

Kenneth Schwartz, MD, FRCPC, Robert Madan MD, FRCPC Baycrest

\section{Learning Objectives:}

1. To understand the benefits of day hospital programs in the treatment of geriatric depression.

2. To describe the specific factors that lead to the positive treatment outcomes.

3. To describe the patient and family experience in this type of program.

Background: Depression in older adults is a major public health concern due to its prevalence, morbidity, chronicity, and health-care cost. Research evaluating the effectiveness of day hospitals for treating geriatric mental disorders has revealed improvement in depression score and a variety of other measures. The goal of this qualitative study was to understand the factors that are associated with the success of this program from the perspectives of the clients and their family members. A further goal was to better understand the experience of the client in this type of treatment program.

Methods: Focus groups were conducted involving clients currently in the day hospital, graduates of the program, and family members of clients. 
Results: Specific factors reported to be important to the success of the program and recovery included reconnecting with meaningful activities, reducing isolation, and finding commonality with others. The therapeutic relationships between the staff and the clients were viewed as a very important factor in recovery. Themes of safety, trust, and belonging emerged. Other factors included the availability of the physicians, psycho-education, and communication with family members. Participants reported valuing the opportunity to learn about oneself, support others, and express feelings in a safe environment.

Conclusions: A day hospital program for the treatment of geriatric depression can be effectively implemented. Aspects of the program such as feelings of safety, trust, and belonging contribute heavily to its success. Communication, education, flexibility, and strong therapeutic relationships were also important elements.

\section{Part 2: A Community Outpatient Model for the Treatment of Depression: the Baycrest Psychiatric Day Hospital Intervention Training Manual}

Kenneth Schwartz, MD, FRCPC, Robert Madan MD, FRCPC, Judi Cohen, MSW, RSw

Baycrest

\section{Learning Objectives:}

1. To help in the development of effective treatment goals for depressed older adults, which assumes greater importance with an ever-increasing and underserviced aging population struggling to cope with multiple losses and psychological stressors.

2. To disseminate the clinical knowledge and expertise that the Baycrest Psychiatric Day Hospital has accumulated in over 25 years of operation.

3. To assist other health-care professionals in developing, organizing, and implementing a similar and effective multicomponent and multi-disciplinary psychiatric day hospital or an intensive outpatient community treatment program.

Background: Given the extraordinary current and projected growth of our elderly population and the relationship between wellness, perceived isolation, and social engagement, ${ }^{(1)}$ the use of groups and group therapy is indicated with this population. The depressed elderly are often socially isolated and without adequate social supports.

Methods: The Baycrest Psychiatric Day Hospital (PDH) uses integrated and cognitive-behavioural group and individual therapies, along with various other group treatments and antidepressant medications. Marital and/or family therapy are employed when appropriate. Treatments occur over four months in a therapeutic social milieu comprised of 20 patients and interdisciplinary staff. Weekly follow-up psychotherapy groups are offered.

Results: The Psychiatric Day Hospital for Depression at Baycrest Centre opened in 1986. Effectiveness is demonstrated by its longevity, waiting list for admission, and the results of outcome studies. ${ }^{(2,3,4)}$ Case illustrations demonstrating the combined use of medications, psychotherapies, and social support of the milieu will allow the participants to see how an intensive community outpatient program can contribute to system reduction, improved quality of life, and even personal growth in depressed other adults. ${ }^{(5)}$

Conclusions: Elderly adults require additional treatment considerations because of medical co-morbidity, cognitive decline, lack of social support, adverse responses to medications, and polypharmacy. ${ }^{(6)}$ The Baycrest PDH provides more comprehensive treatment than routine outpatient therapy in a setting that is less intensive, less expensive, and more community-focused than in-patient care.

1. Cornwell EY, Waite LJ. Social disconnectedness, perceived isolation, and health among older adults. J Health Soc Behavior. 2009;50(1):31-48.

2. Conn DK, Clarke D, van Reekum R. Depression in holocaust survivors: profile and treatment outcome in a geriatric day hospital program. Int J Geriatr Psychiatry. 2000;15(4):331-37.

3. Mackenzie CS, Rosenberg M, Major M. Evaluation of a psychiatric day hospital program for elderly patients with mood disorders. Int Psychogeriatr. 2006;18(4):631-41.

4. Vine RG, Steingart AB. Personality disorders in the elderly depressed. Can J Psychiatry. 1994;39(7):392-98.

5. Schwartz KM, Madan RA. Community outpatient model for the treatment of depression: the Baycrest Psychiatric Day Hospital Intervention Training Manual. Toronto: Baycrest; 2011.

6. Blazer DG. Depression in late-life: review and commentary. $J$ Gerontol Med Sci. 2003;58A(3):249-65.

\section{POSTER ABSTRACTS}

Evaluation of a Symptom-Triggered Protocol

Approach to the Management of Alcohol

P1. Withdrawal Syndrome in the Geriatric

Population

Peter Chan, MD, FRCPC, Asal Taheri, BScPharm, Karen Dahri, PharmD, Amneet Aulakh, PharmD, Maureen Shaw, RN Vancouver General Hospital

\section{Learning Objectives:}

1. To recognize differences in managing alcohol withdrawal in medically ill vs. non-medically ill populations.

2. To discuss a modified symptom-triggered alcohol withdrawal monitoring and treatment protocol in those aged 70 or over. 
3. To know the challenges that manifest with implementing a new protocol in an acute care medical unit.

Background: Compared to their younger counterparts, the geriatric population is at higher risk for alcohol withdrawal associated complications. There are no studies that have evaluated a symptom-triggered approach to management of alcohol withdrawal syndrome (AWS) with benzodiazepine therapy in this population.

Methods: A retrospective health record review was conducted in a large teaching hospital in Vancouver, Canada. Patients 70 years and older were included who, from 2008 to 2012, were admitted to the Acute Care for Elders and Clinical Teaching Unit wards with diagnostic codes for AWS. In 2010 and beyond, a symptom-triggered CIWA-protocol tailored for age 70 or over was implemented for all those suspected of being at risk for alcohol withdrawal. Measured outcomes included median duration and cumulative dose of benzodiazepine treatment, number of severe AWS complications, severe benzodiazepine associated adverse effects, and need for adjunct therapy.

Results: A total of 33 patients in the pre-protocol group and 30 patients in the post-protocol group met the inclusion criteria. Compared to the pre-protocol period, the median duration of benzodiazepine treatment decreased from 96 hours (IQR 72-120) to 48 hours (IQR 0-108) ( $p=.043$ ), and the median cumulative benzodiazepine dose administered reduced from $9 \mathrm{mg}$ (IQR 5-19.8) to $3 \mathrm{mg}$ (IQR $0-10)(p=$ $.001)$. Statically significant lower incidence of severe AWS complications $(p=.007)$ and adjunct therapy use $(p=.017)$ was seen in the protocol-implemented group. CIWA-Ar monitoring was fully adhered to, as dictated by the protocol, in only $40 \%$ of patients though, as one limitation to this study. A nursing survey ( $n=15)$ outlined the challenges.

Conclusions: A symptom-triggered protocol for dosing of benzodiazepine therapy in the management of AWS in adults 70 years and older significantly reduced the total duration of benzodiazepine use, cumulative benzodiazepine dose, and use of adjunctive medications in the treatment of AWS

Exploring Visual Attention as a Biomarker for

P2. Working Memory in Patients with Alzheimer's Disease

Sarah Chau, $\mathrm{MSc}, \operatorname{PhD}(\mathrm{C})^{1,2}$, Nathan Herrmann, MD, $\operatorname{FRCP}(C)^{2,3}$, Moshe Eizenman, $\mathrm{PhD}^{4}$, Larry Grupp, DSc ${ }^{1}$, Krista Lanctot, $\mathrm{PhD}^{1,2}$ ${ }^{1}$ Department of Pharmacology/Toxicology, University of Toronto; ${ }^{2}$ Sunnybrook Health Sciences Centre; ${ }^{3}$ Faculty of Medicine, University of Toronto; ${ }^{4}$ Institute of Biomaterials $\&$ Biomedical Engineering, University of Toronto

\section{Learning Objectives:}

1. Be familiar with the impact of attention deficits in Alzheimer's disease.

2. Be aware of the potential of eye-tracking technology in improving understanding of cognitive deficits in Alzheimer's disease.

3. Be aware of the potential of eye-tracking technology as a reliable, direct, non-verbal biomarker of working memory.

Background: Alzheimer's disease (AD) is associated with impairments in attention, which could underlie many of the deficits in cognition and function. The objective of this study is to examine the attention patterns of AD patients using a visual attention scanning technology (VAST) in order to explore working memory and attention.

Methods: This is a cross-sectional study of mild-to-moderate AD patients and elderly controls. Participants viewed slides containing four images simultaneously presented. Two images on each slide are novel and two are repeats of images that were shown previously; we are testing repeats of $1(n=1)$ and 2 slides back $(n=2)$. The outcome measure was time spent on specific images on a slide (relative fixation time [RFT]). Working memory and attention was also evaluated with the Wechsler Digit Span backward subtest (DSB).

Results: 44 AD patients (24 males, age $=77.6 \pm 8.5$, MMSE $=21.8 \pm 3.9)$ and 26 controls $(9$ males, age $=71.4 \pm 8.9$, $\mathrm{MMSE}=28.2 \pm 2.1)$ were included in this analysis. A twoway ANOVA revealed significant main effects of disease $(\mathrm{F} 1,136=24.6, p<.001)$, image type $(\mathrm{F} 1,136=66.7, p<.001)$, and an interaction $(\mathrm{F} 1,136=7.2, p<.01)$ for RFT in the 1-back condition. Similar results were found in the 2-back condition: disease (F1,136=14.3, $p<.001)$, image type (F1,136=6.3, $p$ $<.05)$, and an interaction $(\mathrm{F} 1,136=6.8, p=.05)$. Significant correlations were found between RFT on repeated images and DSB in the 1-back $(r=0.30, p<.001, n=70)$ and 2-back $(r=0.43, p<.001, n=70)$ conditions.

Conclusions: These preliminary data suggest that orientation towards novel stimuli observed in cognitively intact subjects was not observed in AD patients. The VAST n-back paradigm may be a reliable biomarker of working memory.

Treatment Algorithm for Agitation and

Aggression in Alzheimer's and Mixed Dementia

Simon JC Davies, DM, MBBS, MRCPsych, MSc

Geriatric Psychiatry Division, CAMH; University of Toronto

\section{Learning Objectives:}

1. To recognize the implications of BPSD symptoms (behavioural \& psychological symptoms of dementia) such 
as agitation and aggression for the care of people with dementia, such as Alzheimer's and Mixed Dementia.

2. To be able to list some of the evidence-based, nonpharmacological and pharmacological strategies used to treat BPSD symptoms of agitation and aggression.

3. To appreciate that non-pharmacological treatments include purposeful social contact (e.g., pet therapy) sensory enhancement, purposeful activity (helping roles), and physical activity (e.g., walks), while atypical antipsychotics (e.g., risperidone) are considered first-line drug treatment.

Background: A multidisciplinary team consisting of psychiatrists, nurses, occupational and recreational therapists, and managers was convened to devise an evidence-based treatment pathway for agitation and aggression in BPSD (behavioural and psychological symptoms of dementia) for people with Alzheimer's or Mixed Dementia in hospital in-patient settings.

Methods: Design involved two treatment segments, a nonpharmacological section to which all authors contributed, and a pharmacological section designed primarily by the psychiatrists.

Results: The pathway begins with a phase of assessment of symptoms using standard rating instruments, followed by withdrawal of any medications that might be contributing to the problem. Non-pharmacological treatments include purposeful social contact (e.g., pet therapy), sensory enhancement, purposeful activity (e.g., helping roles), physical activity (e.g., walks), and neurocognitive intervention technology. Pharmacological treatment consists of stepwise trials with the following medications; risperidone, aripiprazole or quetiapine, carbamazepine, citalopram, gabapentin, and prazosin (and subsequently ECT). Titration schedules are provided for each drug with adjustments for frailty. Additional guidance is provided on optimization of existing cholinesterase inhibitors/memantine treatment, and PRN medications, including trazodone and benzodiazepines. Standard assessments are repeated regularly to examine the impact of treatments and assist decisions on whether to continue the current treatment or switch to the next.

Conclusion: This algorithm-based approach for treatment of agitation and aggression in BPSD in the in-patient setting has the potential to allow better outcomes (e.g., faster symptom control, decreased length-of-stay, and higher caregiver and patient satisfaction). The pathway has assessment measures built-in, which will allow for audit of its effectiveness.

Mental Health Nurses: Gatekeepers for an

P4. Inter-professional Geriatric Community Mental Health Team

\section{Learning Objectives:}

1. Members of an inter-professional geriatric mental health team will recognize the value of each discipline and work in tandem in an effort to foster the best possible outcome for the referred patient and their family.

Background: As our population ages, there is an increasing need for seniors' mental health services. Increase in demand and finite financial and human resources require a collaborative approach within inter-professional mental health teams and also with community partners, family/caregivers, and the senior referred to the service.

Methods: Our Inter-professional Geriatric Mental Health Community Team, comprised of professionals with complementary skills, strives to provide a service that helps seniors toward optimum health. Registered Nurses, specialized in geriatric mental health, are the gatekeepers to accessing the service/team. Registered Nurses triage all new referrals for degree of urgency and appropriateness and this helps to insure ease of access in a timely fashion. Once accepted, the referral source is notified of the acceptance and the referred senior is contacted usually within 7-14 days. A Registered Nurse completes an initial assessment. This includes a semi-structured interview and the completion of standardized cognitive screening. The comprehensive bio-psycho-social-cultural assessment is then completed by a Geriatric Psychiatrist in collaboration with the Registered Nurse, the person, and family member/caregiver. When required, other members of the Geriatric Mental Health Community Team, including a Neuropsychologist, Occupational Therapist, and Social Worker, may provide their specialized service. Input is solicited from team members, the referred person, family, and community agencies and then a treatment plan that is ever-evolving is established collaboratively.

Results: Self-determination is key in the recovery process, recognizing that persons directing their own care and making choices are critical components in the collaborative care model that this community team has established.

Conclusions: This community-based team, largely comprised of mental health nurses, collectively believes that the journey of collaboration, culture, and change starts with the person/senior.

Response without Remission with Right

P5. Unilateral Ultrabrief Pulse ECT: Switch to Brief

Pulse Unilateral ECT can be Effective. A Report of Two Cases

Holly Dornan, MD, FRCPC, Lisa McMurray, MD, FRCPC University of Ottawa/ Royal Ottawa Mental Health Centre
Dianne Dillon-Samson, RN, CPMHN(C), GNC(C)

Brockville Mental Health Centre 


\section{Learning Objectives:}

1. To review current evidence in the literature of efficacy and tolerability of right unilateral brief pulse and ultrabrief pulse widths in electroconvulsive therapy (ECT).

2. To present two cases of patients with major depressive disorder who responded, but did not remit, to an initial course of right unilateral ultra-brief pulse ECT.

3. To discuss clinical strategies for managing partial responders.

Background: Electroconvulsive therapy (ECT) is one of the most effective treatments in psychiatry. New research involving different electrode placements and pulse widths has allowed us to be more precise in giving effective ECT while minimizing cognitive side effects. However, controversy exists in the ECT community over the preferred technique. Presently, there are relatively few randomized controlled trials to guide practitioners in their choices.

Methods: We describe two cases of geriatric patients with major depressive disorder who achieved a response, but not remission, with a course of right unilateral ultra-brief pulse (RUL-UB) ECT, as evidenced by a plateau in the depression scores (measured using the Montgomery-Åsberg Depression Rating Scale (MADRS)) after 10 and 12 treatments of RUL-UB ECT.

Results: In both instances, the patients achieved complete remission in their depressive symptoms following a switch to right unilateral brief pulse (RUL-B) ECT. For both of the patients, there was no evidence of cognitive adverse effects with either pulse width. A switch to bilateral ECT was not required, therefore allowing both patients to attain remission while preserving the cognitive advantages of the right unilateral electrode placement.

Conclusions: These cases add to an emerging literature surrounding the decreased efficacy of RUL-UB ECT. We propose that a personalized, algorithm-driven approach to choosing electrode placement and pulse width could lead to better patient outcomes and clinical care.

The Flipped Classroom and Social Media:

P6. Experiences with a Geriatric Psychiatry Unit

in Undergraduate Medical Education

Michelle Gibson, MD, MEd, CCFP, Melissa Andrew, MD, MEd Queen's University

\section{Learning Objectives:}

1. To review an example of a flipped classroom approach, integrating a variety of online and in-class techniques, used for undergraduate geriatric teaching.

2. To become familiar with a model for incorporating Twitter use in geriatric psychiatry teaching.
3. To consider ways in which social media may be utilized to enhance student engagement in the learner's own teaching contexts.

Background: Geriatric psychiatry undergraduate teaching at Queen's occurs at the end of two pre-clinical years. To enhance student engagement, we introduced a flipped classroom model using online modules and team-based learning (TBL) sessions, during which we actively encouraged Twitter use.

Methods: We designed two online modules for students to complete prior to class: a narrated presentation on "approach to delirium", and an interactive module, which includes videos, cognitive testing results, and investigation reports, guiding an approach to cognitive impairment. Using a preselected hashtag, students were encouraged to live-tweet during the in-class sessions. At breaks, Visible Tweets ${ }^{\mathrm{TM}}$ displayed the Twitter discussion. Teachers monitored the feed between and after the sessions, and responded to student tweets.

Results: Over $90 \%$ of students accessed the online resources prior to class. Approximately $15 \%$ of students live-tweeted the session. Teachers observed a high level of engagement in class. Student exam performance indicated good understanding of material, especially on a short-answer question that required significant conceptual integration.

Conclusions: The flipped classroom approach was effective for teaching geriatric topics. Students engaged well with the online resources, and were able to apply principles to cases presented in class and on the exam. Familiarity of this cohort with the flipped classroom model may have enhanced learning. As students were also discerning regarding the execution of a flipped classroom, their positive feedback was particularly meaningful. The fact that learning sessions were well-established was key to success of the intervention, and Twitter simply supported the learning for students who wished to engage in social media. Schizophrenia: A Pilot Study

Angela C. Golas, MD

Department of Psychiatry, University of Toronto

\section{Learning Objectives:}

1. To assess the tolerability and efficacy of CR on cognitive performance in older patients with schizophrenia.

2. To assess the effectiveness of CR on functional competence.

Background: Cognitive deficits are among the strongest predictors of function in individuals with schizophrenia. No pharmacological interventions reliably improve these impairments. As patients grow older, additional age-related 
declines are observed. A number of studies have shown that cognitive remediation (CR) improves cognition in patients with schizophrenia. To date, the efficacy and tolerability of $\mathrm{CR}$ in the geriatric patient population with schizophrenia remains to be studied.

Methods: CR consists of weekly therapist-guided sessions involving didactic teaching, computerized drills targeting different neurocognitive domains, in-class strategic monitoring, and bridging of cognitive skills to daily life. Drills are practiced during the week to consolidate the skills learned in class. Assessments occur at baseline and end-of-study. Clinical symptom severity is assessed using the PANSS; cognition is assessed using a battery of tests, including the MATRICS Consensus Cognitive Battery.

Results: Thirteen participants completed CR in three cohorts (mean age $=70.8, \mathrm{SD}=5.8$, female $=8$ ). Baseline Global Cognition t-scores for cognition ranged from 16-43 (mean $=27.5, \mathrm{SD}=8)$. End-of-study t-scores for Global Cognition ranged from 19-45 $($ mean $=29.8, \mathrm{SD}=9)$. Global cognition improved significantly after CR (paired $\mathrm{t}(12)=2.231, p=$ $.034, d=0.76)$. Clinical symptom severity did not change after CR. To optimize adherence to exercise practice between training sessions, paper-based exercises were substituted with computer lab access for the second cohort, targeting 200 minutes/week of online practice. Mean time spent practicing online exercises ranged from $38-235 \mathrm{~min} /$ week (mean $=111, \mathrm{SD}=61$ ).

Conclusions: The introduction of a computer lab for practice during the week resulted in increased adherence and the ability to monitor performance by the research team. CR is being modified to optimize a) the degree of improvement in the cognitive domains tested, and b) exercise performance and adherence. We are adapting the computer hardware, software program, and the computer lab ergonomics to better meet needs of the geriatric population.

Resident Needs and Attitudes Towards Geriatric

P8. Psychiatry: Results of a Pan-Canadian Online Survey of Psychiatry Residents

Vincent Laliberte, $\mathrm{MD}, \mathrm{MSc}^{1}$, Soham Rej, MD ${ }^{1}$, Mark Rapoport, MD, FRCPC ${ }^{2}$, Melissa Andrew, MD, MEd, FRCPC ${ }^{3}$, Marla Davidson, $\mathrm{MD}, \mathrm{FRCPC}^{4}$

${ }^{1}$ McGill University; ${ }^{2}$ University of Toronto; ${ }^{3}$ Queen's University; ${ }^{4}$ University of Saskatchewan

\section{Learning Objectives:}

1. To learn about an online survey of Canadian psychiatry residents that examined predictors of interest in geriatric psychiatry.

2. To understand the methodology and results of this online survey.
3. To reflect on future strategies to improve recruitment and training of psychiatry residents in geriatric psychiatry.

Background: In spite of a pending "silver tsunami", there remains a shortage of Canadian geriatric psychiatrists. The demographic, educational, and vocational predictors of residents' interest in geriatric psychiatry have not yet been clearly elucidated.

Methods: We conducted a Canada-wide Web-based crosssectional survey, which aimed to ascertain what percentage of Canadian psychiatry residents are interested in: a) becoming geriatric psychiatrists, b) obtaining FRCPC geriatric psychiatry certification, and c) working with older adults as part of their practice. Bivariate and multivariate analyses were performed to better understand what demographic, educational, and vocational variables are associated with interest in becoming a geriatric psychiatrist.

Results: 207/853 (24.3\%) of all psychiatry residents responded to the survey. $29 \%$ of respondents $(n=60)$ had either an interest or a strong interest in becoming a geriatric psychiatrist. Bivariate analyses revealed that although demographic factors were not associated with interest in geriatric psychiatry, certain educational variables (e.g., early and positive training experiences in geriatric psychiatry), and vocational factors (e.g., enjoyment of working with complex multi-factorial clinical issues, including physical health issues) were important predictors of interest. Multivariate analyses will be completed by mid-April 2014 and will be ready for CAGP 2014.

Conclusions: The results of this study may help inform psychiatry residency programs, geriatric psychiatry fellowship programs, the Canadian Academy of Geriatric Psychiatry (CAGP), and other stakeholders when implementing strategies to address the shortage of geriatric psychiatrists in Canada.

Cholinesterase Inhibitor Discontinuation in

P9. Patients with Alzheimer's Disease: a

Meta-Analysis of Randomized Controlled Trials

Graham Mazereeuw, $\mathrm{MSc}^{1,2}$, Krista Lanctôt, $\mathrm{PhD}^{1,2}$, Jordana O'Regan, $\mathrm{MSc}^{2}$, Nathan Herrmann, MD FRCP(C) $)^{1,2}$

${ }^{1}$ University of Toronto; ${ }^{2}$ Sunnybrook Research Institute

\section{Learning Objectives:}

1. To learn about the effects of cholinesterase inhibitor discontinuation.

Background: Cholinesterase inhibitors (ChEIs) are recommended for the symptomatic treatment of Alzheimer's disease (AD). Though ChEIs offer modest cognitive benefits, it is unclear for how long benefits persist, and when discontinuation is warranted. This meta-analysis aimed to examine the cognitive effects of ChEI discontinuation in randomized 
double-blind, placebo-controlled studies (RCTs) including patients with mild, moderate, and severe AD.

Methods: Literature was searched using MEDLINE, Embase, PsycInfo, Cochrane Library, Allied and Complementary Medicine Database (AMED), and Cumulative Index to Nursing and Allied Health Literature (CINAHL) up to March 2014. Trial methods and outcomes were extracted. Meta-analytic methods using fixed-effects models summarized the effects of ChEI discontinuation on cognition (MMSE), behaviour (neuropsychological inventory [NPI]), and adverse events.

Results: Five ChEI discontinuation RCTs, comprising 321 patients continued on a ChEI and 332 patients discontinued from ChEI use, met inclusion criteria. Discontinued patients demonstrated a significant worsening of both cognition (standard mean (SM) MMSE difference: 0.29 [95\% CI 0.13 to $0.45], N=300 / 307, p<.001$ ), and neuropsychiatric symptoms (standard mean NPI difference: -0.32 [95\% CI -0.51 to -0.12], $N=199 / 211, p=.001$ ) compared to patients who continued. No difference in adverse events was observed (OR 1.01 [95\% CI 0.85 to 1.20$], N=314 / 326, p=.92$ ).

Conclusions: The results of this meta-analysis suggest that ChEI discontinuation was associated with significant worsening of cognition and neuropsychiatric symptoms. However, more studies are needed in order to provide clinicians with guidance about when it is safe and appropriate to discontinue treatment.

Mindfulness-Oriented Interventions:

P10. Are They Beneficial for Older and Younger Adult Psychiatric In-patients?

Katerina Nikolitch, $\mathrm{MD}^{1}$, Natalie Strychowsky, MOT $^{2}$, Vincent Laliberte, MD ${ }^{1}$, Ching Yu, MD ${ }^{1}$, Karl Looper, MD, FRCPC ${ }^{1,2}$, Marilyn Segal, MD, FRCPC ${ }^{1,2}$, Soham Rej, MD ${ }^{1}$

${ }^{1}$ McGill University; ${ }^{2}$ Jewish General Hospital

\section{Learning Objectives:}

1. Be able to identify specific in-patient subpopulations (including geriatric populations) who would benefit from mindfulness therapy.

2. Be able to identify subcomponents of mindfulness therapy that are more suitable for the specific in-patient subpopulations.

Background: Mindfulness has been reported to have positive impact on patients' overall well-being; however, little is known about in-patient and severely mentally-ill populations. We aim to identify 1) which older and younger adult in-patients are suitable for mindfulness-oriented therapies, and 2) which types of mindfulness-oriented interventions are most beneficial for these patients.
Methods: Using retrospective data from a mixed-age sample of psychiatric in-patients above and below age 60, we will examine potential predictors of being able to benefit from and tolerate a number of group-therapy, mindfulness-oriented interventions (imaginal relaxation, mindful eating, tai-chi). The primary outcome measure is "change in well-being (or stress) during the intervention". Regression analyses will be performed using a number of potential predictor variables including age, gender, main psychiatric diagnosis, functional status, and a number of mindfulness-related variables.

Results: Data from approximately 100 patients will be analyzed and the results will be presented at the CAGP conference.

Conclusions: We expect mindfulness therapy interventions to be beneficial in an important subgroup of psychiatry inpatients. The demographic and clinical characteristics of these patients may help guide the future development of mindfulness interventions for older and younger adults with severe mental illness.

Short-latency Afferent Inhibition of the

P11. Dorsolateral Prefrontal Cortex in

Healthy Subjects

Yoshihiro Noda, $\mathrm{MD}, \mathrm{PhD}^{1}$, Robin Cash, $\mathrm{PhD}^{2}$, Robert Chen, $\mathrm{MD}$, FRCPC $^{2}$, Zafiris Daskalakis, MD, PhD, FRCPC ${ }^{1}$, Daniel Blumberger, $\mathrm{MD}, \mathrm{MSc}, \mathrm{FRCPC}^{1}$

${ }^{1}$ Centre for Addiction and Mental Health; ${ }^{2}$ Toronto Western Hospital

\section{Learning Objectives:}

1. To learn the importance of cholinergic function in healthy and Alzheimer's disease.

2. To learn short-latency afferent inhibition (SAI) that is a neurophysiological index of cholinergic function in the brain.

3. To learn characteristics of SAI in the dorsolateral prefrontal cortex (DLPFC) in healthy subjects.

Background: Short latency afferent inhibition (SAI) is a promising a neurophysiological test that measures cortical cholinergic activity in healthy and diseased states. However, previous studies of SAI in Alzheimer's disease are limited in two important ways. First, the measure was limited to the motor cortex, a brain region not typically associated with the pathophysiology of this disorder. Second, the aforementioned studies did not evaluate the link between SAI and cognitive function. In this study we aimed to evaluate SAI as a neurophysiological marker of cortical cholinergic activity in brain structures more closely associated with the pathophysiology of $\mathrm{AD}$ (i.e., dorsolateral prefrontal cortex [DLPFC]).

Methods: We measured SAI from the DLPFC by using a new technique of combined electroencephalography (EEG) 
recording during transcranial magnetic stimulation (TMS) in 12 healthy control subjects. To confirm SAI of the DLPFC, we explored at ISIs, that is between median nerve and cortical stimulation, of the latency of $0, \mathrm{~N} 20, \mathrm{~N} 20+2, \mathrm{~N} 20+4, \mathrm{~N} 20+6$, $\mathrm{N} 20+8, \mathrm{~N} 20+10$, and $\mathrm{N} 20+20$.

Results: We observed SAI in the DLPFC at ISIs from N20 to $\mathrm{N} 20+10$ at the N100 component. The strongest SAI was demonstrated at ISI of N20 in the DLPFC.

Conclusions: This is a first study to investigate SAI in the DLPFC, and the measure of SAI in the DLPFC could potentially enhance our understanding of neurophysiology in healthy and diseased states and facilitate the detection of early pathophysiological changes in $\mathrm{AD}$, in a non-invasive and inexpensive way.

Health Care Experiences in Primary Care:

P12. Perspectives of Community-Dwelling Persons with Dementia and their Caregivers

Jeanette Prorok, $\mathrm{MSc}^{1}$, Salinda Horgan, $\mathrm{PhD}^{2}$, Dallas Seitz, $\mathrm{MD}^{1}$ ${ }^{1}$ Providence Care Mental Health Services, Queen's University; ${ }^{2}$ University of Ontario Institutes of Technology

\section{Learning Objectives:}

1. Participants will learn of an existing framework describing the stages of the health-care experience for persons with dementia and their caregivers, and associated health service implications.

2. Participants will learn of challenges experienced by persons with dementia and their caregivers throughout the diagnosis and management processes.

3. Participants will learn of how existing challenges and gaps in care experiences could be addressed to improve care provided to persons with dementia and their caregivers.

Background: Dementia diagnosis and management guidelines recommend that primary care providers play a prominent role in dementia care. However, persons with dementia and their caregivers have expressed several challenges in their experiences with diagnosis and management of dementia in primary care. This study used focus groups conducted with community-dwelling persons with dementia and their caregivers to explore these health-care experiences.

Methods: Participants were recruited through local Alzheimer Society Chapter support groups. Focus group questions were developed as a guide. The focus groups were audio-recorded, transcribed verbatim, and anonymized. The transcripts were reviewed and coded for themes independently by two members of the research team. Data were analyzed within the context of an existing published framework of the health-care experiences of persons with dementia and their caregivers.
Results: Five focus groups were conducted across urban and rural settings in Eastern Ontario. The focus groups ranged in duration from 71 to 86 minutes, with a mean duration of 80 minutes. Each focus group included both persons with dementia and their caregivers. Eight persons with dementia and 21 caregivers participated in the five focus groups. Several themes emerged, including the typically long-winded road to diagnosis, supports, and services, as well as the need for the persons with dementia or caregivers to 'push' for diagnosis, referrals, and supports.

Conclusions: The health-care experiences of persons with dementia and their caregivers are complex. Understanding these experiences may help to improve care, as well as existing supports and services.

P13. Optimizing Responsive Behaviours in Senior-Friendly Acute Care Hospitals

Kiran Rabheru MD, CCFP, FRCP

The Ottawa Hospital

\section{Learning Objectives:}

1. Consider the impact of responsive behaviours in patients with dementia who are in an acute hospital setting.

2. Consider strategic initiatives to improve patient flow and reduce alternate level of care bed utilization in an acute care setting.

3. Improve patient safety and quality of care in acute hospital settings for dementia patients with responsive behaviours.

Background: Dementia is a major public health priority and a key diagnosis in utilization of Alternate Level of Care (ALC) beds. One in five patients have responsive behaviours. Change is imperative in the acute care setting to provide timely access, and prevent ED, ALC, and home care congestion. A complex and resilient hospital-based solution, building on the current processes and focused on reducing utilization of ALC and improving flow through the ED is offered.

Methods: In 2010, the Ministry funded the first phase of an Ontario Behavioural Support System Project (BSS). In March 2013, with strong advocacy and support from the Champlain LHIN, approval received for a Geriatric Psychiatry Behavioural Support Team at TOH. The $\$ 240 \mathrm{~K}$ provided for two RNs. The key intervention for this team will be integration with the four pillars of the Champlain LHIN BSS Action Plan, including 1) System Coordination and Management, 2) Integrated Service Delivery, 3) Knowledge Exchange and Capacity Building, nad 4) Strong Linkages with all key partners.

Results: Evaluation Parameters:

1. Access to service

2. Effectiveness of service 
3. Appropriateness of Service

4. Academic impact

5. Patient safety

6. Enhanced Patient / family / community experience

Conclusions: Creation of a senior-friendly, comprehensive, collaborative In-patient and Emergency Department Behavioural Support Program at TOH, to meet enhanced safety standards and best practices. Future Directions: Scaling Up-A Behavioral Support Program is a new initiative within acute care hospitals, and it is anticipated that this successful project will be adapted for wider use throughout Ontario and Canada. Team for Dementia Persons in Acute Care

Dianne Rossy, RN, BN, MScN, GNC(C), Kiran Rabheru MD, CCFP, FRCP, Jodi Lusk, RN, BScN, Vera Hula, RN

The Ottawa Hospital

\section{Learning Objectives:}

1. Describe the evaluation metrics for implementation of a new Geriatric Psychiatry Behavioural Support Ontario (BSO) team for acute care.

2. Relate the determinants of the program development including inclusion and exclusion criteria and supportive documentation.

3. Review the results of implementation and lessons learned.

Background: There are currently Behavioural Support Ontario (BSO) funded initiatives for persons with dementia and challenging behaviours in long-term care and in the community. In 2014, the Champlain LHIN funded the first pilot BSO Team for acute care at The Ottawa Hospital. This pilot program is a partnership between Geriatric Psychiatry (GP) and Geriatrics, and is a supporting strategy that includes ALC LOS, readmission rates of those presenting with dementia, and emergency room aversion.

Methods: The BSO was implemented in October 2014. Two registered nurses with specialized training are available by consult. The referring unit is cued to meet the criteria for the consult population through the consult form and must ensure the staff physician is in agreement. This then indicates a medical referral to the GP physician. The BSO nurse expert reviews the case, interviews the patient, designs a care plan, and reviews this with the unit staff and the GP physician. The GP physician will also assess and implement interventions for approximately $90 \%$ of the patients. Follow-up and transitions in care are monitored. An online Recording Log sheet records activity and indicators.

Results: The first six months of data support a re-admission rate of $<25$ per cent. Transitions in care are enhanced by a referral rate of $25 \%$ of patients to supporting BSO programs post-discharge. Case load is maintained at approximately 10-15 cases per month. Metrics for 2015 will be shared.

Conclusions: This program is successful in implementation, increasing a monthly referral rate, and in highlighting a vulnerable dementia population in acute care that is traditionally under-reported.

Recherche Évaluative sur la satisfaction des médecins de famille par rapport aux services

P15. offerts par le Service de gérontopsychiatrie du Pavillon Albert-Prévost de l'HSCM, dans le cadre du Plan d'Action en Santé Mentale (PASM)

Majda Ines Senouci, MD ${ }^{1}$, Nathalie Shamlian, MD FRCPC $^{2}$, Anne Décary, $\mathrm{PhD}^{2}$

${ }^{1}$ Université de Montréal; ${ }^{2}$ Hôpital du Sacré-Coeur de Montréal

\section{Learning Objectives:}

1. Élaborer des stratégies pour offrir des soins partagés plus spécifiques aux besoins d'un vaste bassin de population.

2. Évaluer la satisfaction des médecins de famille par rapport à notre travail de collaboration.

3. Partager avec nos collègues, lors du congrès, une organisation de soins spécifique à une clinique de deuxième et troisième ligne en gérontopsychiatrie, qui travaille en continuité avec la première ligne.

Background: Pluralité de modèles de soins partagés, ce qui témoigne d'un travail de collaboration et d'une structure de soins qui demeurent très spécifique à la population visée et à l'organisation locale des soins de santé. De nombreuses études se sont penchées sur la pratique de soins partagés en psychiatrie adulte, mais très peu concernent les soins dédiés à la population gériatrique. Les patients nécessitant des soins en santé mentale constituent une population particulièrement vulnérable. Cette réalité est d'autant plus vraie dans un contexte gériatrique, où les patients nécessitent des soins complexes, multidisciplinaires et s'inscrivant dans une continuité avec la première ligne.

Methods: Lettre et questionnaire distribués aux 131 médecins de famille travaillant en première ligne dans le CSSS Bordeau-Cartierville-Saint-Laurent, à Montréal; appels aux médecins et à leurs secrétaires pour les mettre au courant du projet; projet annoncé dans le journal interne de l'HSCM; lettres et enveloppes préaffranchies remises aux secrétaires dans les différentes cliniques (T0).

Results: Les GP se disent globalement assez satisfaits de notre travail de collaboration $(4.4 / 5 \pm 0.19)$ et disent nous référer des patients à l'avenir très souvent (4.7/5 \pm 0.11$)$. Les consultations répondent aux questions et aident à la prise 
en charge dans la majorité des cas $(4.6 / 5 \pm 0.11$ et $4 / 5 \pm 0.31)$. La prise en charge par notre équipe est jugée généralement adéquate $(4 / 5 \pm 0.31)$. Le délai d'attente est jugé plutôt satisfaisant (3.44/5 \pm 0.25$)$. Sur 9 ayant répondu à la question, 5 disent préférer une feuille synthèse résumant la consultation au rapport complet. Difficile de préciser les besoins d'une population quand l'échantillonnage est restreint.

Conclusions: Dans l'avenir, pistes de travail: a) L'accès à une expertise psychiatrique spécifique à une population gériatrique demeure très appréciée de nos collègues de la première ligne; b) Des besoins grandissants d'une expertise en soins partagés et de collaboration en gérontopsychiatrie se font ressentir par nos collègues de première ligne; c) Nécessité de développer des stratégies de soins partagés spécifiques à la gérontopsychiatrie; d) Éventuellement, définition des rôles à préciser dans le prochain PASM.

\section{Medical Predictors of Psychiatric}

P16. Re-hospitalization in Older Patients with Severe Mental Illness

Jean-Daniel Sylvestre, $\mathrm{MD}^{1}$, Ching Yu, $\mathrm{MD}^{1}$, Marilyn Segal, MD, FRCPC $^{1,2}$, Karl Looper, MD, FRCPC ${ }^{1,2}$, Soham Rej, MD ${ }^{1}$

${ }^{1}$ McGill University; ${ }^{2}$ Jewish General Hospital

\section{Learning Objectives:}

1. To be able to determine the medical characteristics that can predict frequent psychiatric re-hospitalization.

2. To discuss possible strategies to target the specific patient population who are more susceptible to psychiatric re-hospitalization.

Background: Many patients with severe mental illness are approaching late life. In this population, we expect a high prevalence of medical co-morbidities, which may contribute to serious consequences, including frequent psychiatric re-hospitalization. Our objectives are to quantify the prevalence and correlates of frequent re-hospitalization in geriatric psychiatric in-patients, and to identify medical characteristics that can predict frequent psychiatric re-hospitalization (i.e., chronic medical illnesses, baseline renal functions).

Methods: This is a retrospective study of geriatric psychiatric patients admitted to a tertiary care Canadian in-patient psychiatric unit between 2003 and 2008. We have ascertained the incidence of psychiatric re-admission in the subsequent 5-year period (e.g., 2008-2013 if a patient had been first admitted in 2008). We then examined whether medical variables (lab values at index admission, chronic medical conditions, and renal functions) would predict frequent psychiatric re-hospitalization.
Conclusions: We expect a high prevalence of frequent psychiatric re-hospitalization in our sample. The medical characteristics of these patients may help guide future intervention to prevent re-hospitalization (e.g., home visits).

\section{P17. Geriatric Psychiatry Clients:} Barriers to Discharge

Lisa VanBussel, $\mathrm{FRCPC}^{1,2,3}$, Shane Reed, BSW, Rsw ${ }^{1,2}$, Julie Smith, BSw, RSw ${ }^{1,2}$, Jack Li ${ }^{1,2}$, Ed Black, PhD CPsych ${ }^{1,2}$

${ }^{1}$ St. Joseph's Health Care, London; ${ }^{2}$ Regional Mental Health Care, London; ${ }^{3}$ Western University, London

\section{Learning Objectives:}

1. Awareness of barriers to discharging patients.

2. Use of barriers to discharge form to support clinical decision-making.

3. Reviewing root causes of prolonged hospitalization.

Background: This quantitative evaluation examined barriers to timely discharge from an in-patient Geriatric Psychiatry Tertiary setting.

Methods: In-patient cases identified what resources are required to transition clients from the hospital setting. The barriers to discharge summary consisted of four main factors: patient, family, system, and long term care (LTC). Under each factor, specific barriers were identified, and each patient was categorized according to their perceived barriers. If clients remain in hospital after 45 days, a social worker completed the barriers form and updated the form every 90 days. Residential Assessment Instrument (RAI) was used to measure activities of daily living, cognitive function, pain, aggression, and depression.

Results: Findings indicate multiple factors contribute to barriers to discharge. The population included $62.5 \%$ males. Frequency of barriers: patient related $=101, \mathrm{LTC}=51$, system $=25$, and family $=23$. Overall, the most frequent specific barrier was inadequate symptom control. There were barrier differences between the long-term stay (LTS, $>1$ year stay) and Short Term Stay (STS, $<1$ year stay) clients. The LTS group mainly consisted of clients suffering from dementia with no clients suffering from mood disorder, as opposed to the STS group which was more evenly distributed between clients suffering from dementia, schizophrenia, and mood disorder. The STS group had no Alternate Level of Care (ALC) clients, while the LTS included 6 ALC clients.

Conclusions: These results support the use of this instrument to help determine root causes of prolonged hospitalization.

Results: 259 in-patients' data will be analyzed and the results will be presented at the conference. 
Biopsychosocial Predictors of Psychiatric

P18. Re-hospitalization in Older Patients with Severe Mental Illness

Ching Yu, MD ${ }^{1}$, Soham Rej, MD ${ }^{1}$, Marilyn Segal, MD, FRCPC ${ }^{1,2}$, Karl Looper, MD, FRCPC ${ }^{1,2}$, Jean-Daniel Sylvestre, MD ${ }^{1}$

${ }^{1}$ McGill University; ${ }^{2}$ Jewish General Hospital

\section{Learning Objectives:}

1. To determine the psychiatric clinical and demographic characteristics that can predict frequent psychiatric rehospitalization.

2. To discuss possible strategies to target the specific patient population who are more susceptible to psychiatric rehospitalization.

Background: Many patients with severe mental illness are approaching late life. In this population, non-compliance and other factors may have serious consequences including frequent psychiatric re-hospitalization. Our objectives are to quantify the prevalence and correlates of frequent re-hospitalization in geriatric psychiatric in-patients, and to identify clinical and demographic characteristics that can predict frequent psychiatric re-hospitalization.

Methods: This is a retrospective study of geriatric psychiatric patients admitted to a tertiary care Canadian in-patient psychiatric unit between 2003 and 2008. We have ascertained the incidence of psychiatric re-admission in the subsequent 5 -year period (e.g., 2008-2013 if a patient had been first admitted in 2008). We then examined whether psychiatric clinical and demographic variables (primary psychiatric diagnosis, biopsychosocial characteristics) would predict frequent psychiatric re-hospitalization.

Results: 259 in-patients' data will be analyzed and the results will be presented at the conference.

Conclusions: We expect a high prevalence of frequent psychiatric re-hospitalization in our sample. The demographic and clinical characteristics of these patients may help guide future intervention to prevent re-hospitalization (e.g., home visits).
Apolipoprotein E2 Allele Promotes Spatial

P19. Strategies and Hippocampal Grey matter in a Human Virtual Navigation Task

Venkat Bhat, MD, Kyoko Konishi, MSc, Judes Poirier, PhD, Ridha Joober, MD PhD, Veronique Bohbot, PhD

McGill University

\section{Learning Objectives:}

1. Appreciate that multiple memory systems are involved in human navigation.

2. In healthy young adults, spatial learners have greater proportion of Apolipoprotein E2 allele, and have more grey matter in the hippocampus.

3. Apolipoprotein E2 may be protective against Alzheimer's disease through increased use of spatial strategies.

Background: The Apolipoprotein E gene (ApoE) has been implicated in Alzheimer's disease (AD) and age-related memory decline. This study examines the role of ApoE in spatial memory and choice of navigational strategies among healthy adults.

Methods: 125 healthy adult subjects ( 18 to $35 \mathrm{yrs}$ ) were tested on the 4-on- 8 virtual maze task (4/8VM) and structural MRI scans were obtained. Participants were categorized as response learners or spatial learners based on initial spontaneous strategy, and into three groups based on $\mathrm{ApoE}$ genotypes (E3/ E3, $n=71$; E3/E4, $n=30$; E3/E2, $n=18$ ). Chisquared analysis and ANOVA were used to compare the genotype groups for navigational strategies, demographic, neuropsychological, and neuroimaging parameters.

Results: The ApoE genotype groups significantly differed in their use of navigational strategies $\left(\mathrm{X}^{2}=7.91, \mathrm{df}=2, p<\right.$ $.001)$, with greater of spatial strategy (61\%) in the ApoE3/E2 group, and response strategy (71\%) in the ApoE3/4 group. The MRI results showed that spatial learners have more grey matter in the hippocampus than response learners $(t=2.44, p$ $<.001$ ), and that ApoE2 carriers had significantly more grey matter in the hippocampus than ApoE4 $(t=5.34, p<.001)$.

Conclusions: This is the first study to report role of ApoE in healthy young adults, and suggests that ApoE 2 may be protective against $\mathrm{AD}$ through increased spatial memory strategy use and hippocampal function. This has important therapeutic implications, given that ApoE4 increases risk for Alzheimer's, ApoE2 is protective against it, and spatial memory has a protective effect against onset of dementia. 